\title{
Versatile Reactivity and Theoretical Evaluation of Mono- and Dinuclear Oxidovanadium(V) Compounds of Aroylazines: Electrogeneration of Mixed-Valence Divanadium(IV,V) Complexes
}

\author{
Subhashree P. Dash, ${ }^{\dagger,}$ Satabdi Roy, ${ }^{\dagger}$ Monalisa Mohanty, ${ }^{\dagger}$ M. Fernanda N. N. Carvalho, ${ }^{\S}$ \\ Maxim L. Kuznetsov, ${ }^{\S}$ João Costa Pessoa, ${ }^{*}$, Amit Kumar, $^{\S}, \|$ Yogesh P. Patil, ${ }^{\perp}$ Aurélien Crochet, ${ }^{\mathbb{I I}}$ \\ and Rupam Dinda* ${ }^{* \dagger}$
${ }^{\dagger}$ Department of Chemistry, National Institute of Technology, Rourkela 769008, Odisha, India
${ }^{\ddagger}$ Department of Chemistry, Indira Gandhi Institute of Technology, Sarang, Parjang, Dhenkanal 759146, India
${ }^{\S}$ Centro de Química Estrutural, Instituto Superior Técnico, Universidade de Lisboa, Avenida Rovisco Pais, 1049-001 Lisboa, Portugal
"Centre for Nano and Material Sciences, Jain University, Jain Global Campus, Jakkasandra Post, Bangalore 562112, India
${ }^{\perp}$ Department of Inorganic and Physical Chemistry, Indian Institute of Science, Bangalore 560012, India
${ }^{\mathrm{I}}$ Department of Chemistry, Fribourg Center for Nanomaterials, University of Fribourg, CH-1700 Fribourg, Switzerland

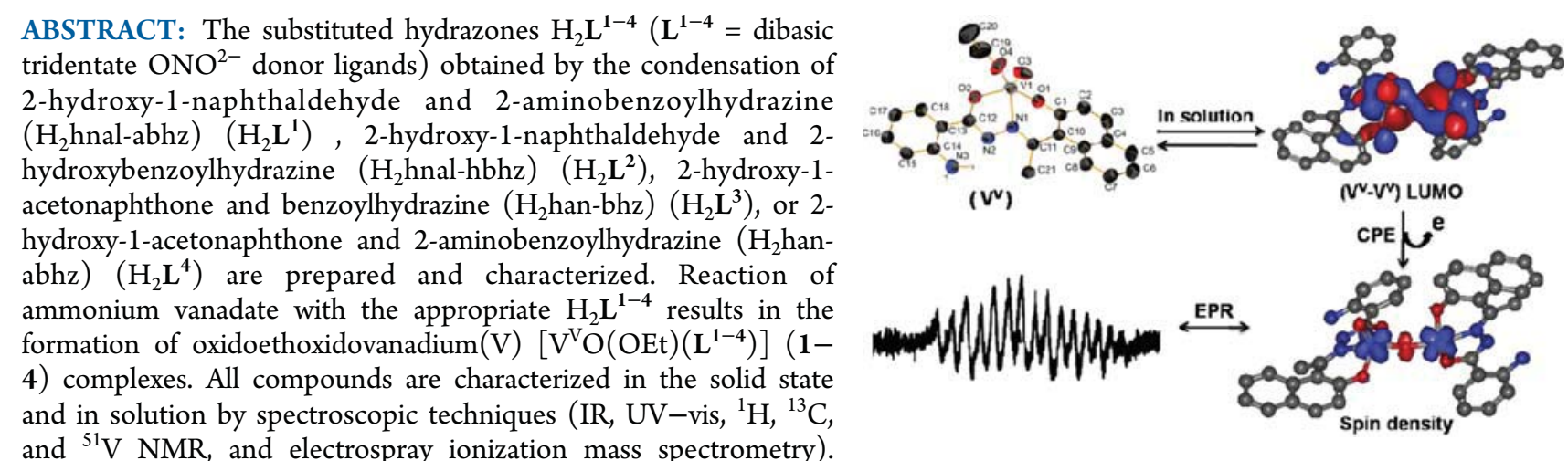
and ${ }^{51} \mathrm{~V}$ NMR, and electrospray ionization mass spectrometry).

Single-crystal X-ray diffraction analysis of $\mathbf{1}, \mathbf{3}$, and $\mathbf{4}$ confirms the coordination of the corresponding ligands in the dianionic $\left(\mathrm{ONO}^{2-}\right)$ enolate tautomeric form. In solution, the structurally characterized $\left[\mathrm{V}^{\mathrm{V}} \mathrm{O}(\mathrm{OEt})(\mathrm{L})\right]$ compounds transform into the monooxido-bridged divanadium $(\mathrm{V}, \mathrm{V})\left[\left(\mathrm{V}^{\mathrm{V}} \mathrm{OL}\right)_{2}-\mu-\mathrm{O}\right]$ complexes, with the processes being studied by $\mathrm{IR}$ and ${ }^{1} \mathrm{H}$, ${ }^{13} \mathrm{C}$, and ${ }^{51} \mathrm{~V}$ NMR. The density functional theory (DFT) calculated Gibbs free energy of reaction $2\left[\mathrm{~V}^{\mathrm{V}} \mathrm{O}(\mathrm{OEt})\left(\mathrm{L}^{4}\right)\right]+\mathrm{H}_{2} \mathrm{O} \leftrightarrows\left[\left(\mathrm{V}^{\mathrm{V}} \mathrm{OL}{ }^{4}\right)_{2}-\mu-\right.$ $\mathrm{O}]+2 \mathrm{EtOH}$ is only $2-3 \mathrm{kcal} \mathrm{mol}^{-1}$, indicating that the dinuclear complexes may form in a significant amount. The electrochemical behavior of the complexes is investigated by cyclic voltammetry, with the $\mathrm{V}^{\mathrm{V}}-\mathrm{V}^{\mathrm{IV}} E_{1 / 2}$ red values being in the range 0.27-0.44 V (vs SCE). Upon controlled potential electrolysis, the corresponding $(\mathrm{L})(\mathrm{O}) \mathrm{V}^{\mathrm{IV}}-\mathrm{O}-\mathrm{V}^{\mathrm{V}}(\mathrm{O})(\mathrm{L})$ mixed-valence species are obtained upon partial reduction of the $\left[\left(\mathrm{V}^{\mathrm{V}} \mathrm{OL}\right)_{2}-\mu-\mathrm{O}\right]$ complexes formed in solution, and some spectroscopic characteristics of these dinuclear mixed-valence complexes are investigated using DFT calculations and by electron paramagnetic resonance (EPR), with the formation of $\mathrm{V}^{\mathrm{IV}}-\mathrm{O}-\mathrm{V}^{\mathrm{V}}$ species being confirmed by the observation of a 15 -line pattern in the EPR spectra at room temperature.

\section{INTRODUCTION}

Interest in the coordination chemistry of vanadium, with particular emphasis on its biological, ${ }^{1}$ structural, ${ }^{1 \mathrm{a}, \mathrm{b}, \mathrm{d}, 2}$ and catalytic properties, ${ }^{3-6}$ has increased over the past 2 decades. Prospective therapeutic applications of vanadium compounds, namely, for the treatment of Diabetes mellitus and cancer and more recently promising antiamoebic activity against Entamoeba histolytica as well as antitrypanosomal activity against Trypanosoma cruzi, ${ }^{1 \mathrm{c}, \mathrm{e}}$ stimulated research to better understand the behavior of vanadium complexes in solution. ${ }^{7-10}$
Vanadium complexes possess versatile oxidation states (from - III to $+\mathrm{V})$. Among these, $+\mathrm{IV}$ and $+\mathrm{V}$ are the most common and can be accessed easily under aerobic conditions. A good number of structural, spectroscopic, and solution studies of such vanadium compounds, in which the element is present in these high oxidation states under assorted N/O-donor environments, have been reported. ${ }^{8 a-c, g, 11}$ 
Scheme 1. Schematic Diagram of the Synthesis of Monooxidoalkoxidovanadium $(\mathrm{V})\left[\mathrm{V}^{\mathrm{V}} \mathrm{O}(\mathrm{OEt})\left(\mathrm{L}^{1-4}\right)\right](1-4)$ and the Various Pathways through Which the Compounds May Be Transformed in Solution

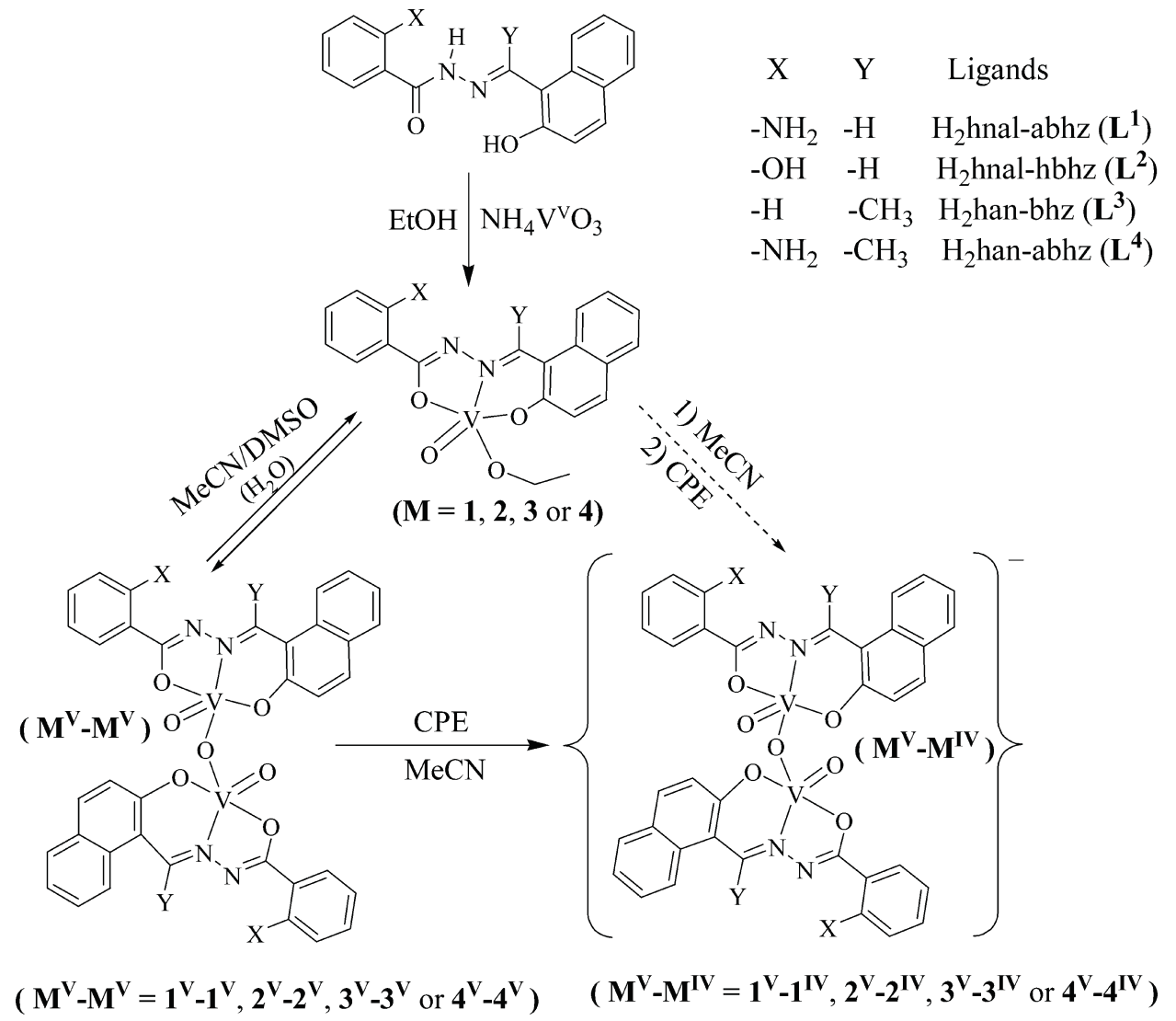

While the solid-state structural aspects and reactivity of the well-known vanadium $(\mathrm{V})$ complexes containing $\mathrm{V}^{\mathrm{V}} \mathrm{O}_{2}{ }^{+}$units have been satisfactorily exposed, not so much is known of their solution behavior. ${ }^{1 \mathrm{~b}, 12}$ Subjects of interest include the stability of mononuclear species in solution, possibility of formation of coordination isomers, ${ }^{12 a, e}$ formation of $\mathrm{di}^{10,12 b, c}$ or polynuclear complexes, ${ }^{12 \mathrm{~d}}$ and electron-transfer capabilities of mono- and dinuclear species. ${ }^{10,12 \mathrm{~b}}$

Mixed-valence metal complexes have received much attention, ${ }^{13}$ and one of the relevant aspects in this area includes an understanding of the role of the ligand environment in the extent of electron localization/delocalization among the metal centers and the potential use of mixed-valence systems in molecular electronics and molecular computing. ${ }^{14}$ The chemistry of mixed-valence vanadium incorporating $\mathrm{O} / \mathrm{N}$ coordinating ligands is of enduring interest. ${ }^{15}$ In contrast to the well-known oxidovanadium(IV or $\mathrm{V}$ ) complexes containing $\mathrm{V}^{\mathrm{IV}} \mathrm{O}^{2+}$ or $\mathrm{V}^{\mathrm{V}} \mathrm{O}^{3+}$ units, ${ }^{15 \mathrm{~b}, 16}$ relatively few mixed-oxidation $(\mathrm{IV}, \mathrm{V})$ complexes have been isolated and structurally characterized. In the context of the present study, it is relevant to mention that, although the chemistry of mixed-valence divanadium $(\mathrm{IV}, \mathrm{V})$ complexes generated through chemical synthesis is reasonably developed, ${ }^{15,17}$ there are only a few reported examples of compounds isolated through electrosynthesis. ${ }^{12 \mathrm{~b}, 15 \mathrm{~d}, 18}$

We describe herein the synthesis and characterization of mononuclear monoalkoxido-bound monooxidovanadium(V) complexes $(1-4)$ of the ligands derived from the substituted hydrazones $\mathrm{H}_{2} \mathrm{~L}^{1-4}\left(\mathrm{H}_{2} \mathrm{~L}^{1-4}=\right.$ aroylhydrazones, where $\mathrm{L}^{1-4}$ act as dibasic tridentate ONO-donor ligands; Scheme 1); these ligands may be considered as azine analogues. We also show that the transformation of the structurally characterized monooxidoalkoxidovanadium $(\mathrm{V})$ compounds $\left[\mathrm{V}^{\mathrm{V}} \mathrm{O}(\mathrm{OEt})(\mathrm{L})\right]$ in solution lead to the corresponding monooxido-bridged divanadium $(\mathrm{V}, \mathrm{V})$ compounds $\left[\left(\mathrm{V}^{\mathrm{V}} \mathrm{OL}\right)_{2}-\mu-\mathrm{O}\right] .{ }^{12 \mathrm{~b}}$ The monooxido-bridged divanadium $(\mathrm{IV}, \mathrm{V})$ mixed-valence species are also obtained by controlled potential electrolysis of the corresponding divanadium $(\mathrm{V}, \mathrm{V})$ precursors in solution. These mixedvalence species are characterized by IR, electron paramagnetic resonance (EPR), and ${ }^{51} \mathrm{~V} \mathrm{NMR}$, and their formulation was corroborated by density functional theory (DFT) calculations. Theoretical calculations were also carried out for most complexes reported to support and substantiate the measured structural and spectroscopic properties. We should highlight that, in agreement with Young, ${ }^{15 a}$ we are designating here, as mixed-valence $\mathrm{V}_{2} \mathrm{O}_{3}{ }^{3+}$ compounds, both those that correspond to complexes with localized $\mathrm{V}^{\mathrm{IV}}$ and $\mathrm{V}^{\mathrm{V}}$ centers, exhibiting 8line EPR spectra, and those where the unpaired electron is delocalized throughout the two $\mathrm{V}$ nuclei, exhibiting a 15-line EPR spectra due to the effective hyperfine coupling of the two ${ }^{51} \mathrm{~V}$ centers. It is demonstrated that spin localization/ delocalization takes place between two $\mathrm{V}$ atoms, and, hence, a different pattern of EPR spectra for various compounds (15line vs 8-line) is determined by the geometry of the $\mathrm{V}-\mathrm{O}-\mathrm{V}$ fragment (linear vs bent).

\section{EXPERIMENTAL SECTION}

Materials and Methods. Elemental analyses were carried out on a PerkinElmer $\mathrm{C}, \mathrm{H}$, and $\mathrm{N}$ analyzer. IR spectra of solid compounds $\left[\mathrm{V}^{\mathrm{V}} \mathrm{O}(\mathrm{OEt})(\mathrm{L})\right](\mathbf{1 - 4})$ as $\mathrm{KBr}$ pellets and in a $\mathrm{CHCl}_{3}$ solution in a $\mathrm{NaCl}$ cell were recorded on a PerkinElmer 783 spectrometer. ${ }^{1} \mathrm{H}$ 
NMR spectra were recorded with a Bruker ADVANCE DPX $300 \mathrm{MHz}$ spectrometer using $\mathrm{SiMe}_{4}$ as an internal standard. ${ }^{51} \mathrm{~V}$ NMR spectra were obtained on a Bruker Avance III $400 \mathrm{MHz}$ spectrometer in deuterated acetonitrile:dimethylsulfoxide $\left(\mathrm{MeCN}-d_{1}: \mathrm{DMSO}-d_{6}\right)$, and chemical shift ${ }^{51} \mathrm{~V}$ values $\left(\delta^{\mathrm{V}}\right)$ were referenced relative to neat $\mathrm{V}^{\mathrm{V}} \mathrm{OCl}_{3}$ as the external standard. Mass spectrometry (MS) spectra were obtained on a SQ-300 MS instrument operating in the electrospray ionization (ESI) negative mode. Electronic spectra were recorded on a Shimadzu UV-vis recording spectrophotometer. Electrochemical studies were made by cyclic voltammetry $(\mathrm{CV})$ and controlled potential electrolysis (CPE) at room temperature under a nitrogen atmosphere using a PC-controlled VoltaLab PST 050 potentiostat. Measurements were made in a three-compartment cell provided with platinum wire (working and auxiliary) and silver wire (reference) electrodes using tetrabutylammonium tetrafluoroborate $(0.10 \mathrm{M}) /$ $\mathrm{CH}_{3} \mathrm{CN}$ as the supporting electrolyte. Potentials were referred to a saturated calomel electrode (SCE) using the ferrocene/ferrocenium couple $\left(\left[\mathrm{Fe}\left(\eta^{5}-\mathrm{C}_{5} \mathrm{H}_{5}\right)_{2}\right]^{0 /+} ; E_{1 / 2}=0.382 \mathrm{~V}\right)$ as the internal standard. Electron paramagnetic resonance (EPR) spectra were recorded with a Bruker ESP $300 \mathrm{E} X$-band spectrometer either at $77 \mathrm{~K}$ or at room temperature, and the spin Hamiltonian parameters were obtained by simulation of the spectra with the computer program of Rockenbauer et al. ${ }^{19}$ The calibrant was 2,2-diphenyl-1-picrylhydrazyl ( $g$ : 2.0037).

Crystal Structure Determinations. Single crystals of compounds were mounted on a Bruker Smart Apex CCD diffractometer (1) equipped with a graphite monochromator and a Stoe IPDS 2 diffractometer (3 and 4) equipped with an Oxford Cryosystem openflow cryostat and a Mo K $\alpha$ radiation $(\lambda=0.71073 \AA)$. The crystallographic data and details of refinement are given in Table 1. The unit cell dimensions and intensity data were measured at 293(2) $\mathrm{K}(1)$ and 200 (2) K (3 and 4). Absorption correction was partially integrated in the data reduction procedure for crystals of 3 and $4 .^{20}$ The intensity data were corrected for Lorentz, polarization, and absorption effects. Absorption corrections were applied using $S A D A B S$, and the structures were solved by direct methods using the program SHELXS- $97^{21}$ and refined using least squares with the SHELXL- $97^{21}$ software program. $\mathrm{H}$ atoms were either found or placed in calculated positions and isotropically refined using a riding model. The non- $\mathrm{H}$ atoms were refined anisotropically.

CV and CPE. The redox properties of complexes 1-4 were studied by $\mathrm{CV}$, and the potentials were measured versus SCE using ferrocene as the internal standard. CPE was carried out at the potential of the reduction process (selected according to $\mathrm{CV}$ data) using a typical procedure: the addition of a weighed amount of complex [1 $(5.0 \mathrm{mg})$, $2(5.0 \mathrm{mg}), 3(7.0 \mathrm{mg})$, and $4(8.0 \mathrm{mg})]$ to the electrolyte solution (10 $\mathrm{mL} ; \mathrm{NBu}_{4} \mathrm{BF}_{4} / \mathrm{CH}_{3} \mathrm{CN}, 0.10 \mathrm{M}$, under a nitrogen atmosphere), waiting ca. $1 \mathrm{~h}$, and then proceeding with the chronocoulometric process until it reached the number of coulombs $(Q)$ corresponding to transfer of half an electron per mole of complex. Then, a cyclic voltammogram was obtained, a sample of the electrolyzed solution was collected and immediately transferred to an EPR tube (previously purged with nitrogen gas), and the solution was frozen by emersion of the tube in liquid nitrogen.

Computational Details. Full geometry optimization of the molecular structures was carried out at the DFT level of theory using the B3P86 functional ${ }^{22 a}$ with the help of the Gaussian09 program package. $^{23}$ This functional was found to be sufficiently accurate for theoretical studies of structural parameters and ${ }^{51} \mathrm{~V}$ NMR chemical shifts of various vanadium complexes. ${ }^{24}$ For some calculations, B3LYP, ${ }^{22 a, c} M_{06}{ }^{22 \mathrm{~d}}$ and TPSSH ${ }^{22 \mathrm{e}}$ functionals were also used. Optimization was carried out in both gas and solution phases. In the latter case, the IEFPCM solvation model ${ }^{25}$ with the UAKS molecular cavity and dispersion, repulsion, and cavitation contributions were applied, and DMSO, ethanol $(\mathrm{EtOH})$, or $\mathrm{MeCN}$ were used as the solvent. No symmetry operations were applied for any of the structures calculated. Geometry optimization was carried out using a relativistic Stuttgart pseudopotential, which describes 10 core electrons, and the appropriate contracted basis set (8s7p6d1f)/ [6s5p3dif] for the $\mathrm{V}$ atom ${ }^{26}$ and the $6-31 \mathrm{G}(\mathrm{d})$ basis set for other atoms. The Hessian matrix was calculated analytically for all optimized
Table 1. Crystal Data and Refinement Details for 1, 3, and 4

\begin{tabular}{|c|c|c|c|}
\hline & 1 & 3 & 4 \\
\hline empirical formula & $\mathrm{C}_{20} \mathrm{H}_{18} \mathrm{~N}_{3} \mathrm{O}_{4} \mathrm{~V}$ & $\mathrm{C}_{21} \mathrm{H}_{19} \mathrm{~N}_{2} \mathrm{O}_{4} \mathrm{~V}$ & $\mathrm{C}_{21} \mathrm{H}_{20} \mathrm{~N}_{3} \mathrm{O}_{4} \mathrm{~V}$ \\
\hline$M_{\mathrm{w}}$ & 415.31 & 414.32 & 429.34 \\
\hline temperature $(\mathrm{K})$ & $293(2)$ & $200(2)$ & $200(2)$ \\
\hline wavelength $(\AA)$ & 0.71073 & 0.71073 & 0.71073 \\
\hline cryst syst & monoclinic & monoclinic & monoclinic \\
\hline space group & $P 2_{1} / n$ & $P 2_{1} / n$ & $P 2_{1} / n$ \\
\hline unit cell dimens & & & \\
\hline$a(\AA)$ & $7.5992(7)$ & $10.0073(19)$ & $10.305(2)$ \\
\hline$b(\AA)$ & $12.7639(11)$ & $15.0465(18)$ & $10.502(2)$ \\
\hline$c(\AA)$ & $19.5684(17)$ & $13.023(2)$ & $18.603(3)$ \\
\hline$\alpha(\mathrm{deg})$ & 90 & 90 & 90 \\
\hline$\beta(\operatorname{deg})$ & $90.373(2)$ & $91.581(15)$ & $92.716(16)$ \\
\hline$\gamma(\operatorname{deg})$ & 90 & 90 & 90 \\
\hline volume $\left(\AA^{3}\right)$ & $1898.0(3)$ & $1960.2(6)$ & $2011.0(6)$ \\
\hline$Z$ & 4 & 4 & 4 \\
\hline $\begin{array}{l}\text { density (calcd) } \\
\left(\mathrm{Mg} \mathrm{m}^{-3}\right)\end{array}$ & 1.453 & 1.404 & 1.418 \\
\hline abs coeff $\left(\mathrm{mm}^{-1}\right)$ & 0.554 & 0.535 & 0.526 \\
\hline$F(000)$ & 856 & 856 & 888 \\
\hline $\begin{array}{l}\theta \text { range for data } \\
\text { collection (deg) }\end{array}$ & $1.90-26.00$ & $2.07-25.00$ & $2.192-24.995$ \\
\hline reflns collected & 19292 & 24699 & 25250 \\
\hline indep reflns & $\begin{array}{l}3732[R(\text { int })= \\
0.0788]\end{array}$ & $\begin{array}{l}3452[R(\text { int })= \\
0.0843]\end{array}$ & $\begin{array}{l}3549[R(\text { int })= \\
0.1342]\end{array}$ \\
\hline $\begin{array}{l}\text { completeness to } \theta \\
=25.00^{\circ}(\%)\end{array}$ & 100.0 & 100.0 & 100.0 \\
\hline $\begin{array}{l}\text { refinement } \\
\text { method }\end{array}$ & $\begin{array}{l}\text { full-matrix least } \\
\text { squares on } F^{2}\end{array}$ & $\begin{array}{c}\text { full-matrix least } \\
\text { squares on } F^{2}\end{array}$ & $\begin{array}{r}\text { full-matrix least } \\
\text { squares on } F^{2}\end{array}$ \\
\hline $\begin{array}{l}\text { data/restraints/ } \\
\text { param }\end{array}$ & $3732 / 1 / 262$ & $3452 / 48 / 255$ & $3549 / 0 / 265$ \\
\hline GOF on $F^{2}$ & 1.045 & 1.043 & 0.920 \\
\hline $\begin{array}{l}\text { final } R \text { indices }[I> \\
2 \sigma(I)]\end{array}$ & $\begin{array}{l}\mathrm{R} 1=0.0939 \\
\mathrm{wR} 2=0.2142\end{array}$ & $\begin{array}{l}\mathrm{R} 1=0.0382 \\
\mathrm{wR} 2=0.1034\end{array}$ & $\begin{aligned} \mathrm{R} 1 & =0.0497, \mathrm{wR} 2 \\
= & 0.1164\end{aligned}$ \\
\hline$R$ indices (all data) & $\begin{array}{l}\mathrm{R} 1=0.1668 \\
\mathrm{wR} 2=0.2584\end{array}$ & $\begin{array}{l}\mathrm{R} 1=0.0512 \\
\mathrm{wR} 2=0.1091\end{array}$ & $\begin{array}{l}\mathrm{R} 1=0.1224, \mathrm{wR} 2 \\
=0.1521\end{array}$ \\
\hline $\begin{array}{l}\text { largest diff peak } \\
\text { and hole }\left(\mathrm{e} \AA^{-3}\right)\end{array}$ & $\begin{array}{c}0.589 \text { and } \\
-0.319\end{array}$ & $\begin{array}{c}0.230 \text { and } \\
-0.287\end{array}$ & 0.257 and -0.365 \\
\hline
\end{tabular}

structures to prove the location of correct minima (no imaginary frequencies) and to estimate the thermodynamic parameters, with the latter being calculated at $25{ }^{\circ} \mathrm{C}$.

Magnetic shielding was calculated for the equilibrium geometries using the GIAO method ${ }^{27}$ at the IEFPCM-B3P86/6-311+G(2d,p)//6$31 \mathrm{G}(\mathrm{d})$ level. The ${ }^{51} \mathrm{~V}$ chemical shifts were estimated relative to $\mathrm{V}^{\mathrm{V}} \mathrm{OCl}_{3}$ calculated at the same level of theory.

The ${ }^{51} \mathrm{~V}$ hyperfine coupling constants were estimated at the singlepoint calculations using the BHandHLYP functional and $6-311 \mathrm{G}^{*}$ basis set for all atoms on the basis of the equilibrium geometry obtained at the B3P86/6-31G*(V-ECP) level of theory. The anisotropic ${ }^{51} \mathrm{~V}$ hyperfine coupling constants $A_{x}, A_{y}$, and $A_{z}$ were estimated as the sum of the isotropic Fermi contact term and corresponding dipolar hyperfine interaction term. ${ }^{28}$ It must be emphasized that for a vanadium(IV) species the hyperfine coupling constant $(A)$ values may be negative, but in the literature, normally it is their absolute value that is reported. This simplification was also adopted in this work. Previously, it was found that half-and-half functionals such as BHandHLYP demonstrate better performance in calculations of the EPR parameters than many other hybrid functionals because of more accurate estimates of the Fermi contact term. ${ }^{28 a-c}$

For the dinuclear vanadium $(\mathrm{V}, \mathrm{V})$ compounds $\left[\left(\mathrm{V}^{\mathrm{V}} \mathrm{OL}\right)_{2}-\mu-\mathrm{O}\right]$, two isomers were calculated, i.e., the trans isomer with the opposite positions of the $\mathrm{V}=\mathrm{O}$ bonds relative to the $\mathrm{V}-\mathrm{O}-\mathrm{V}$ bridge and the gauche isomer with the $\mathrm{O}=\mathrm{V} \cdots \mathrm{V}=\mathrm{O}$ torsion angle of $78-103^{\circ}$. The latter isomer is thermodynamically more stable, and mainly structures of this type are discussed below. 
Preparation of Compounds $\mathrm{H}_{2} \mathrm{~L}^{1-4}$. The substituted hydrazones $\mathrm{H}_{2}$ hnal-abhz $\left(\mathrm{H}_{2} \mathbf{L}^{1}\right), \mathrm{H}_{2}$ hnal-hbhz $\left(\mathrm{H}_{2} \mathbf{L}^{2}\right)$, and $\mathrm{H}_{2}$ han-bhz $\left(\mathrm{H}_{2} \mathbf{L}^{3}\right)$, and $\mathrm{H}_{2}$ han-abhz $\left(\mathrm{H}_{2} \mathrm{~L}^{4}\right)$ were prepared by similar procedures; we describe here the preparation of $\mathrm{H}_{2} \mathrm{~L}^{1}$ : a solution of 2-aminobenzoylhydrazine $(1.51 \mathrm{~g}, 10.0 \mathrm{mmol})$ in $\mathrm{EtOH}(20 \mathrm{~mL})$ was added to a filtered solution of 2-hydroxy-1-naphthaldehyde $(1.72 \mathrm{~g}, 10.0 \mathrm{mmol})$ in $\mathrm{EtOH}(15 \mathrm{~mL})$ with stirring. The obtained mixture was refluxed and stirred for $2 \mathrm{~h}$. The resulting pale-yellow compounds were filtered, washed with $\mathrm{EtOH}$, and dried over fused $\mathrm{CaCl}_{2}$. Elemental analysis results and NMR $\left({ }^{1} \mathrm{H}\right.$ and $\left.{ }^{13} \mathrm{C}\right)$ and IR data for all compounds corroborate with their formulation.

$\mathrm{H}_{2}$ hnal-abhz $\left(\mathrm{H}_{2} \mathrm{~L}^{1}\right)$. Yield: $65 \%$. Anal. Calcd for $\mathrm{C}_{18} \mathrm{H}_{15} \mathrm{~N}_{3} \mathrm{O}_{2}$ : C, $70.81 ; \mathrm{H}, 4.95 ; \mathrm{N}, 13.76$. Found: C, 70.77; H, 4.98; N, 13.75. Selected IR peaks with proposed assignments $\left(\mathrm{KBr}\right.$ pellet, $\left.\nu_{\max } / \mathrm{cm}^{-1}\right): 3472$ $\nu(\mathrm{O}-\mathrm{H}) ; 3367 \nu\left(\mathrm{NH}_{2}\right)_{\mathrm{as}} ; 3201 \nu\left(\mathrm{NH}_{2}\right)_{s} ; 3037 \nu(\mathrm{N}-\mathrm{H}) ; 1637 \nu(\mathrm{C}=$ O); $1555 \nu(\mathrm{C}=\mathrm{N}) .{ }^{1} \mathrm{H}$ NMR $\left(400 \mathrm{MHz}\right.$, DMSO-d $\left.d_{6}\right): \delta 12.97(\mathrm{~s}, 1 \mathrm{H}$ naphthyl-OH), $11.95(\mathrm{~s}, 1 \mathrm{H}, \mathrm{NH}), 9.47(\mathrm{~s}, 1 \mathrm{H}, \mathrm{HC}=\mathrm{N}), 8.18-6.63$ (m, 10H, aromatic), $6.61\left(\mathrm{~s}, 2 \mathrm{H}, \mathrm{NH}_{2}\right) \cdot{ }^{13} \mathrm{C}$ NMR (100 MHz, DMSO$\left.d_{6}\right): \delta 165.12,158.33,150.93,146.33,133.18,132.91,132.07,129.45$, $128.57,128.25,128.16,123.96,120.88,119.42,117.12,115.14,112.62$, 109.06

$\mathrm{H}_{2}$ hnal-hbhz $\left(\mathrm{H}_{2} \mathrm{~L}^{2}\right)$. Yield: $75 \%$. Anal. Calcd for $\mathrm{C}_{18} \mathrm{H}_{14} \mathrm{~N}_{2} \mathrm{O}_{3}$ : C, 70.58; H, 4.61; N, 9.15. Found: C, 70.59; H, 4.58; N, 9.12. Selected IR peaks with proposed assignments $\left(\mathrm{KBr}\right.$ pellet, $\left.\nu_{\max } / \mathrm{cm}^{-1}\right): 3466 \nu(\mathrm{O}-$ $\mathrm{H}) ; 3022 \nu(\mathrm{N}-\mathrm{H}) ; 1640 \nu(\mathrm{C}=\mathrm{O}) ; 1578 \nu(\mathrm{C}=\mathrm{N}) .{ }^{1} \mathrm{H}$ NMR $(400$ $\left.\mathrm{MHz}, \mathrm{DMSO}-d_{6}\right): \delta 12.74(\mathrm{~s}, 1 \mathrm{H}$, naphthyl-OH $), 12.07(\mathrm{~s}, 1 \mathrm{H}, \mathrm{NH})$ $11.87(\mathrm{~s}, 1 \mathrm{H}$, aryl-OH), $9.54(\mathrm{~s}, 1 \mathrm{H}, \mathrm{HC}=\mathrm{N}), 8.33-6.62(\mathrm{~m}, 10 \mathrm{H}$, aromatic). ${ }^{13} \mathrm{C}$ NMR $\left(100 \mathrm{MHz}\right.$, DMSO- $\left.d_{6}\right): \delta 163.72,158.51,157.89$, $147.44,133.78,132.68,131.44,128.69,128.55,127.57,127.50,123.33$, $120.73,118.92,118.65,117.05,115.48,108.36$.

$\mathrm{H}_{2}$ han-bhz $\left(\mathrm{H}_{2} \mathrm{~L}^{3}\right)$. Yield: $58 \%$. Anal. Calcd for $\mathrm{C}_{19} \mathrm{H}_{16} \mathrm{~N}_{2} \mathrm{O}_{2}$ : C, 74.98; H, 5.30; N, 9.20. Found: C, 74.96; H, 5.32; N, 9.21. Selected IR peaks with proposed assignments $\left(\mathrm{KBr}\right.$ pellet, $\left.\nu_{\max } / \mathrm{cm}^{-1}\right): 3337 \nu(\mathrm{O}-$ $\mathrm{H}) ; 3071 \nu(\mathrm{N}-\mathrm{H}) ; 1659 \nu(\mathrm{C}=\mathrm{O}) ; 1573 \nu(\mathrm{C}=\mathrm{N}) .{ }^{1} \mathrm{H}$ NMR $(400$ $\left.\mathrm{MHz}, \mathrm{DMSO}-d_{6}\right): \delta 10.31$ (s, 1H, naphthyl-OH), $9.55(\mathrm{~s}, 1 \mathrm{H}, \mathrm{NH})$ 7.91-7.27 (m, $11 \mathrm{H}$, aromatic), $2.34\left(\mathrm{~s}, 3 \mathrm{H}, \mathrm{CH}_{3}\right) .{ }^{13} \mathrm{C}$ NMR $(100$ MHz, DMSO- $\left.d_{6}\right): \delta 153.71,152.63,134.24,131.82,131.51,130.21$, $129.06,128.76,128.36,128.23,127.86,127.43,126.99,123.67,123.19$, $118.99,118.71,113.74,24.30$

$\mathrm{H}_{2}$ han-abhz $\left(\mathrm{H}_{2} \mathrm{~L}^{4}\right)$. Yield: $64 \%$. Anal. Calcd for $\mathrm{C}_{19} \mathrm{H}_{17} \mathrm{~N}_{3} \mathrm{O}_{2}$ : C, 71.46; H, 5.37; N, 13.16. Found: C, 71.48; H, 5.37; N, 13.14. Selected IR peaks with proposed assignments $\left(\mathrm{KBr}\right.$ pellet, $\left.\nu_{\max } / \mathrm{cm}^{-1}\right): 3473$ $\nu(\mathrm{O}-\mathrm{H}) ; 3410 \nu\left(\mathrm{NH}_{2}\right)_{\mathrm{as}} ; 3329 \nu\left(\mathrm{NH}_{2}\right)_{\mathrm{s}} ; 3221 \nu(\mathrm{N}-\mathrm{H}) ; 1663 \nu(\mathrm{C}=$ $\mathrm{O}) ; 1553 \nu(\mathrm{C}=\mathrm{N}) .{ }^{1} \mathrm{H}$ NMR $\left(400 \mathrm{MHz}\right.$, DMSO- $\left.d_{6}\right): \delta 10.35(\mathrm{~s}, 1 \mathrm{H}$ naphthyl-OH), $9.17(\mathrm{~s}, 1 \mathrm{H}, \mathrm{NH}), 7.91-6.23(\mathrm{~m}, 10 \mathrm{H}$, aromatic), $6.05\left(\mathrm{~s}, 2 \mathrm{H}, \mathrm{NH}_{2}\right), 2.35\left(\mathrm{~s}, 3 \mathrm{H}, \mathrm{CH}_{3}\right) .{ }^{13} \mathrm{C}$ NMR $(100 \mathrm{MHz}, \mathrm{DMSO}-$ $\left.d_{6}\right): \delta 153.98,152.47,152.36,149.84,132.47,131.56,130.22,129.09$, 128.34, 127.98, 127.67, 123.78, 123.14, 118.94, 117.04, 115.46, 114.69, $113.59,24.22$.

Preparation of $\left[\mathrm{V}^{\mathrm{V}} \mathrm{O}(\mathrm{OEt})(\mathrm{L})\right](1-4)$. We describe here a general synthetic procedure taking $\mathrm{NH}_{4} \mathrm{~V}^{\mathrm{V}} \mathrm{O}_{3}$ as the metal precursor. $\mathrm{NH}_{4} \mathrm{~V}^{\mathrm{V}} \mathrm{O}_{3}(1.0 \mathrm{mmol})$ was added to a hot solution of the appropriate $\mathrm{H}_{2} \mathbf{L}^{1-4}(1.0 \mathrm{mmol})$ in EtOH $(20 \mathrm{~mL})$; the color changed instantly to brown, and after $3 \mathrm{~h}$ of refluxing, the reaction mixture was filtered off and kept for precipitation of the compounds. After 3-4 days, crystals of diffraction quality were obtained and used for X-ray diffraction (XRD) structure determination.

[V $\mathrm{V}$ O(OEt)(hnal-abhz)] (1). Yield: 61\%. Anal. Calcd for $\mathrm{C}_{20} \mathrm{H}_{18} \mathrm{~N}_{3} \mathrm{O}_{4} \mathrm{~V}$ : C, 57.84; H, 4.37; N, 10.12. Found: C, 57.83; H, 4.39; N, 10.08. Selected IR peaks with proposed assignments $(\mathrm{KBr}$ pellet, $\left.\nu_{\max } / \mathrm{cm}^{-1}\right): 3461 \nu\left(\mathrm{NH}_{2}\right)_{\mathrm{as}} ; 3322 \nu\left(\mathrm{NH}_{2}\right)_{\mathrm{s}} ; 1595 \nu(\mathrm{C}=\mathrm{N})$; $1255 \nu(\mathrm{C}-\mathrm{O})_{\text {enolic }} 991 \nu(\mathrm{V}=\mathrm{O})$. IR $\left(\mathrm{CHCl}_{3}, \mathrm{~cm}^{-1}\right): 1002,977(\mathrm{~V}=$ $\mathrm{O}) ; 821(\mathrm{~V}-\mathrm{O}-\mathrm{V})$. UV-vis $\left[\mathrm{CHCl}_{3} ; \lambda_{\max }, \mathrm{nm}\left(\varepsilon, \mathrm{M}^{-1} \mathrm{~cm}^{-1}\right)\right]: 434$ (15257); 336 (19651); 286 (26852); 248 (30614). ESI-MS (DMSO): $m / z 386.16\left(100 \%,\left[\mathrm{~V}^{\mathrm{V}} \mathrm{O}_{2}\left(\mathrm{~L}^{1}\right)\right]^{-}, \mathrm{MW}=386.26\right), 756.13(14 \%$, $\left.\left[\mathrm{V}^{\mathrm{V}} \mathrm{V}^{\mathrm{IV}} \mathrm{O}_{3}\left(\mathrm{~L}^{1}\right)_{2}\right]^{-}, \mathrm{MW}=756.51\right) .{ }^{1} \mathrm{H}$ NMR $\left(400 \mathrm{MHz}, \mathrm{DMSO}-d_{6}\right): \delta$ $9.99(\mathrm{~s}, 1 \mathrm{H}, \mathrm{HC}=\mathrm{N}), 9.94(\mathrm{~s}, 1 \mathrm{H}, \mathrm{HC}=\mathrm{N}), 8.59-6.43(\mathrm{~m}, 20 \mathrm{H}$, aromatic), 5.76-5.54 (m, $\left.2 \mathrm{H}, \mathrm{CH}_{2}(\mathrm{OEt})\right), 4.32\left(\mathrm{~s}, 2 \mathrm{H}, \mathrm{NH}_{2}\right), 3.48-$ $3.43\left(\mathrm{q}, 4 \mathrm{H}, \mathrm{CH}_{2}(\mathrm{EtOH})\right), 1.60-1.57\left(\mathrm{t}, 3 \mathrm{H}, \mathrm{CH}_{3}(\mathrm{OEt})\right), 1.08-1.05$ $\left(\mathrm{t}, 6 \mathrm{H}, \mathrm{CH}_{3}(\mathrm{EtOH})\right) .{ }^{13} \mathrm{C}$ NMR $\left(100 \mathrm{MHz}, \mathrm{DMSO}-d_{6}\right): \delta 171.22$,
170.93, 164.64, 164.46, 164.18, 149.78, 149.65, 148.26, 148.11, 135.71, 135.52, 135.15, 132.91, 132.78, 132.65, 132.41, 130.97, 130.57, 129.44, $129.34,128.82$, 128.54, 128.48, 124.71, 124.35, 122.45, 122.11, 119.90, $119.03,116.39,115.17,111.82,111.67,111.05,110.36,81.80,56.52$, 19.03, 18.84. ${ }^{1} \mathrm{H}$ NMR $\left(400 \mathrm{MHz}\right.$, DMSO- $\left.d_{6}+\mathrm{EtOH}\right): \delta 9.80(\mathrm{~s}, 1 \mathrm{H}$, $\mathrm{HC}=\mathrm{N}), \quad 8.49-6.57(\mathrm{~m}, 10 \mathrm{H}$, aromatic $), 5.73-5.54(\mathrm{~m}, 2 \mathrm{H}$, $\left.\mathrm{CH}_{2}(\mathrm{OEt})\right), 4.33$ (s, 20H, NH$\left.)_{2}\right), 3.49-3.44$ (q, $46 \mathrm{H}, \mathrm{CH}_{2}(\mathrm{EtOH})$ ), $1.60-1.57\left(\mathrm{t}, 3 \mathrm{H}, \mathrm{CH}_{3}(\mathrm{OEt})\right), 1.09-1.05\left(\mathrm{t}, 67 \mathrm{H}, \mathrm{CH}_{3}(\mathrm{EtOH})\right) .{ }^{13} \mathrm{C}$ NMR $\left(100 \mathrm{MHz}, \mathrm{DMSO}-d_{6}+\mathrm{EtOH}\right): \delta 171.06,164.22,149.65$, $148.15,135.04,132.88,132.27,130.54,129.23,128.54,128.39,124.24$, $121.94,119.79,116.31,115.08,111.65,111.07,81.70,56.54,18.75$, 18.64. ${ }^{51} \mathrm{~V}$ NMR (DMSO- $\left.d_{6} / \mathrm{MeCN}-d_{1}(1: 10)\right): \delta-548(85.0 \%)$, $-576(15.0 \%)$.

[V $\left.{ }^{\mathrm{V}} \mathrm{O}(\mathrm{OEt})(\mathrm{hnal}-\mathrm{hbhz})\right]$ (2). Yield: 65\%. Anal. Calcd for $\mathrm{C}_{20} \mathrm{H}_{17} \mathrm{~N}_{2} \mathrm{O}_{5} \mathrm{~V}$ : C, 57.70; H, 4.12; N, 6.73. Found: C, 57.72; H, 4.08; N, 6.77. Selected IR peaks with proposed assignments $(\mathrm{KBr}$ pellet, $\left.\nu_{\max } / \mathrm{cm}^{-1}\right): 3442 \nu(\mathrm{O}-\mathrm{H}) ; 1597 \nu(\mathrm{C}=\mathrm{N}) ; 1254 \nu(\mathrm{C}-$ O) $)_{\text {enolic; }} 996 \nu(\mathrm{V}=\mathrm{O})$. IR $\left(\mathrm{CHCl}_{3}, \mathrm{~cm}^{-1}\right): 1006,980(\mathrm{~V}=\mathrm{O}) ; 823$ $(\mathrm{V}-\mathrm{O}-\mathrm{V})$. UV-vis $\left[\mathrm{CHCl}_{3} ; \lambda_{\max }, \mathrm{nm}\left(\varepsilon, \mathrm{M}^{-1} \mathrm{~cm}^{-1}\right)\right]$ : 425 (12649); 339 (20637); 273 (27392); 245 (27822). ESI-MS (DMSO): $\mathrm{m} / z$ $387.08\left(100 \%,\left[\mathrm{~V}^{\mathrm{V}} \mathrm{O}_{2}\left(\mathrm{~L}^{2}\right)\right]^{-}, \mathrm{MW}=387.02\right), 758.05(30 \%$, $\left[\mathrm{V}^{\mathrm{V}} \mathrm{V}^{\mathrm{VV}} \mathrm{O}_{3}\left(\mathrm{~L}^{2}\right)_{2}\right]-$, MW $\left.=758.48\right) .{ }^{1} \mathrm{H}$ NMR $\left(400 \mathrm{MHz}\right.$, DMSO- $\left.d_{6}\right)$ : $\delta 11.60(\mathrm{~s}, 1 \mathrm{H}, \mathrm{OH}), 11.42(\mathrm{~s}, 1 \mathrm{H}, \mathrm{OH}), 10.09(\mathrm{~s}, 1 \mathrm{H}, \mathrm{HC}=\mathrm{N}), 9.95$ $(\mathrm{s}, 1 \mathrm{H}, \mathrm{HC}=\mathrm{N}), 8.65-6.83(\mathrm{~m}, 20 \mathrm{H}$, aromatic $), 5.86-5.66(\mathrm{~m}, 2 \mathrm{H}$ $\left.\mathrm{CH}_{2}(\mathrm{OEt})\right), 3.47-3.41$ (q, 4H, $\left.\mathrm{CH}_{2}(\mathrm{EtOH})\right), 1.63-1.59(\mathrm{t}, 3 \mathrm{H}$, $\left.\mathrm{CH}_{3}(\mathrm{OEt})\right), 1.07-1.04\left(\mathrm{t}, 6 \mathrm{H}, \mathrm{CH}_{3}(\mathrm{EtOH})\right) .{ }^{13} \mathrm{C} \mathrm{NMR}(100 \mathrm{MHz}$, DMSO- $\left.d_{6}\right): \delta 169.95,164.66,164.29,158.96,158.90,149.52,136.06$, $135.73,133.98$, 133.80, 133.01, 129.72, 129.51, 129.45, 129.34, 128.90, $128.62,124.85,124.53,122.64,122.29,119.76,119.57,118.90,117.31$, $114.69,114.30,111.62,111.46,83.32,56.50,19.02,18.81 .{ }^{1} \mathrm{H}$ NMR $\left(400 \mathrm{MHz}, \mathrm{DMSO}-d_{6}+\mathrm{EtOH}\right): \delta 11.61(\mathrm{~s}, 1 \mathrm{H}, \mathrm{OH}), 9.94(\mathrm{~s}, 1 \mathrm{H}$, $\mathrm{HC}=\mathrm{N}), \quad 8.56-6.97(\mathrm{~m}, 10 \mathrm{H}$, aromatic $), 5.86-5.66(\mathrm{~m}, 2 \mathrm{H}$ $\left.\mathrm{CH}_{2}(\mathrm{OEt})\right), 3.47-3.41\left(\mathrm{q}, 46 \mathrm{H}, \mathrm{CH}_{2}(\mathrm{EtOH})\right), 1.63-1.59(\mathrm{t}, 3 \mathrm{H}$, $\left.\mathrm{CH}_{3}(\mathrm{OEt})\right), 1.07-1.04\left(\mathrm{t}, 68 \mathrm{H}, \mathrm{CH}_{3}(\mathrm{EtOH})\right) .{ }^{13} \mathrm{C} \mathrm{NMR}(100 \mathrm{MHz}$, DMSO- $\left.d_{6}+\mathrm{EtOH}\right): \delta 170.25,164.36,159.04,149.04,135.35,133.31$, $132.92,129.35$, 129.05, 128.61, 128.34, 124.18, 121.80, 119.49, 119.05, $116.95,114.67,111.41,83.14,56.57,18.25 .{ }^{51} \mathrm{~V}$ NMR (DMSO- $d_{6}$ ) MeCN-d $(1: 10)): \delta-554$ (80.0\%), $-594(20.0 \%)$

[V $\left.\mathrm{V}^{\mathrm{O}} \mathrm{O}(\mathrm{OEt})(\mathrm{han}-\mathrm{bhz})\right]$ (3). Yield: 66\%. Anal. Calcd for $\mathrm{C}_{21} \mathrm{H}_{19} \mathrm{~N}_{2} \mathrm{O}_{4} \mathrm{~V}$ : C, 60.88; H, 4.62; N, 6.76. Found: C, 60.86; $\mathrm{H}$, 4.58; N, 6.77. Selected IR peaks with proposed assignments $(\mathrm{KBr}$ pellet, $\left.\nu_{\max } / \mathrm{cm}^{-1}\right): 1595 \nu(\mathrm{C}=\mathrm{N}) ; 1239 \nu(\mathrm{C}-\mathrm{O})_{\text {enolic; }} 999 \nu(\mathrm{V}=\mathrm{O})$. IR $\left(\mathrm{CHCl}_{3}, \mathrm{~cm}^{-1}\right): 1004,966(\mathrm{~V}=\mathrm{O}) ; 820(\mathrm{~V}-\mathrm{O}-\mathrm{V})$. UV-vis $\left[\mathrm{CHCl}_{3} ; \lambda_{\max }, \mathrm{nm}\left(\varepsilon, \mathrm{M}^{-1} \mathrm{~cm}^{-1}\right)\right]: 430$ (7003); 343 (14511); 286 (21713); 234 (25207). ESI-MS (DMSO): $\mathrm{m} / z \quad 385.10$ (64\%, $\left.\left[\mathrm{V}^{\mathrm{V}} \mathrm{O}_{2}\left(\mathbf{L}^{3}\right)\right]^{-}, \mathrm{MW}=385.27\right), 754.19\left(100 \%,\left[\mathrm{~V}^{\mathrm{V}} \mathrm{V}^{\mathrm{IV}} \mathrm{O}_{3}\left(\mathbf{L}^{3}\right)_{2}\right]^{-}, \mathrm{MW}\right.$ $=754.53) .{ }^{1} \mathrm{H}$ NMR $\left(400 \mathrm{MHz}, \mathrm{DMSO}-d_{6}\right): \delta 7.98-7.22(\mathrm{~m}, 22 \mathrm{H}$, aromatic), 5.54-5.41 (m, 2H, $\left.\mathrm{CH}_{2}(\mathrm{OEt})\right), 3.47-3.42(\mathrm{q}, 4 \mathrm{H}$, $\left.\mathrm{CH}_{2}(\mathrm{EtOH})\right), 2.92\left(\mathrm{~s}, 3 \mathrm{H}, \mathrm{CH}_{3}\right), 2.84\left(\mathrm{~s}, 3 \mathrm{H}, \mathrm{CH}_{3}\right), 1.52-1.48(\mathrm{t}$, $\left.3 \mathrm{H}, \mathrm{CH}_{3}(\mathrm{OEt})\right), 1.08-1.04\left(\mathrm{t}, 6 \mathrm{H}, \mathrm{CH}_{3}(\mathrm{EtOH})\right) .{ }^{13} \mathrm{C}$ NMR $(100$ $\left.\mathrm{MHz}, \mathrm{DMSO}-d_{6}\right): \delta 170.91,170.36,166.64,166.47,166.16,160.17$, $134.85,134.75,133.89,132.13,131.82$, 131.77, 130.94, 130.66, 130.01, $129.95,129.56,129.35,128.97,128.70,128.61,128.53,128.00,127.58$, $126.54,126.17,124.99,124.88,124.34,119.50,118.75,118.17,118.03$, $117.86,80.23,56.51,24.09,23.84,19.01,18.67 .{ }^{1} \mathrm{H}$ NMR (400 MHz, DMSO- $\left.d_{6}+\mathrm{EtOH}\right): \delta 8.09-7.20(\mathrm{~m}, 11 \mathrm{H}$, aromatic), 5.54-5.41 $(\mathrm{m}$, $\left.2 \mathrm{H}, \mathrm{CH}_{2}(\mathrm{OEt})\right), 3.48-3.43\left(\mathrm{q}, 56 \mathrm{H}, \mathrm{CH}_{2}(\mathrm{EtOH}), 2.92\left(\mathrm{~s}, 3 \mathrm{H}, \mathrm{CH}_{3}\right)\right.$, $1.53-1.49\left(\mathrm{t}, 3 \mathrm{H}, \mathrm{CH}_{3}(\mathrm{OEt})\right), 1.08-1.04\left(\mathrm{t}, 83 \mathrm{H}, \mathrm{CH}_{3}(\mathrm{EtOH})\right) .{ }^{13} \mathrm{C}$ NMR $\left(100 \mathrm{MHz}\right.$, DMSO- $\left.d_{6}+\mathrm{EtOH}\right): \delta 170.87,166.13,159.73$, $133.80,131.99,131.75,131.69,129.58,129.26,128.86,128.49,127.47$ $126.10,124.25,119.41,118.16,80.20,56.53,23.68,18.77,18.50 .{ }^{51} \mathrm{~V}$ NMR (DMSO- $\left.d_{6} / \mathrm{MeCN}-d_{1}(1: 10)\right): \delta-500(100.0 \%)$.

[V $\mathrm{V}^{\mathrm{O}}(\mathrm{OEt})(\mathrm{han}-\mathrm{abhz})$ ] (4). Yield: 64\%. Anal. Calcd for $\mathrm{C}_{21} \mathrm{H}_{20} \mathrm{~N}_{3} \mathrm{O}_{4} \mathrm{~V}$ : C, 58.75; H, 4.70; N, 9.79. Found: $\mathrm{C}, 58.74 ; \mathrm{H}$, 4.73; N, 9.78. Selected IR peaks with proposed assignments $(\mathrm{KBr}$ pellet, $\left.\nu_{\max } / \mathrm{cm}^{-1}\right): 1567 \nu(\mathrm{C}=\mathrm{N}) ; 1238 \nu(\mathrm{C}-\mathrm{O})_{\text {enolic }} ; 996 \nu(\mathrm{V}=\mathrm{O})$. IR $\left(\mathrm{CHCl}_{3}, \mathrm{~cm}^{-1}\right): 1004,969(\mathrm{~V}=\mathrm{O}) ; 821(\mathrm{~V}-\mathrm{O}-\mathrm{V})$. UV-vis $\left[\mathrm{CHCl}_{3} ; \lambda_{\max }, \mathrm{nm}\left(\varepsilon, \mathrm{M}^{-1} \mathrm{~cm}^{-1}\right)\right]: 419$ (14445); 307 (18634); 277 (24006); 253 (30231). ESI-MS (DMSO): $\mathrm{m} / z \quad 400.02$ (100\%, $\left.\left[\mathrm{V}^{\mathrm{V}} \mathrm{O}_{2}\left(\mathrm{~L}^{4}\right)\right]^{-}, \mathrm{MW}=400.28\right), 784.30\left(50 \%,\left[\mathrm{~V}^{\mathrm{V}} \mathrm{V}^{\mathrm{IV}} \mathrm{O}_{3}\left(\mathrm{~L}^{4}\right)_{2}\right]^{-}, \mathrm{MW}\right.$ 
a)

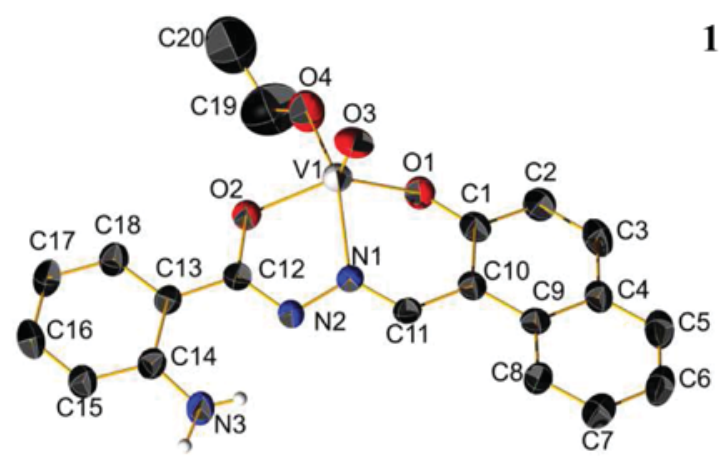

b)

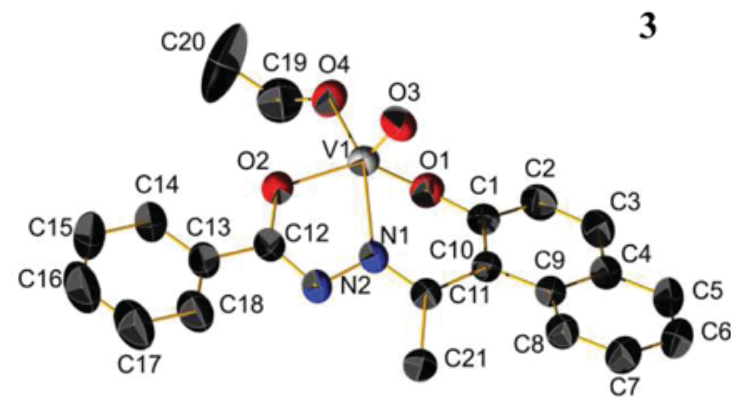

c)

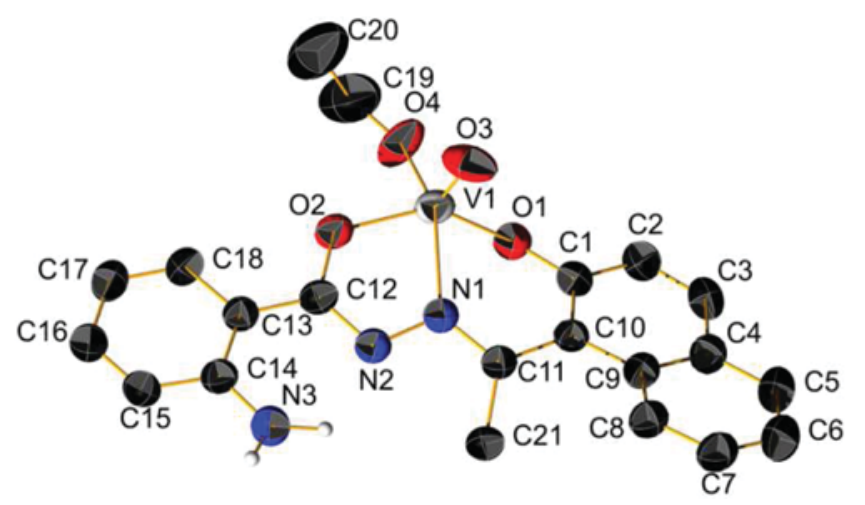

Figure 1. ORTEP (30\%) diagrams of (a) 1, (b) 3, and (c) 4.

$=784.56) .{ }^{1} \mathrm{H}$ NMR $\left(400 \mathrm{MHz}, \mathrm{DMSO}-d_{6}\right): \delta 8.21-6.31(\mathrm{~m}, 20 \mathrm{H}$, aromatic), 5.56-5.42 (m, 2H, $\left.\mathrm{CH}_{2}(\mathrm{OEt})\right), 4.52\left(\mathrm{~s}, 2 \mathrm{H}, \mathrm{NH}_{2}\right), 3.50-$ 3.45 (q, 4H, $\left.\mathrm{CH}_{2}(\mathrm{EtOH})\right), 2.95\left(\mathrm{~s}, 3 \mathrm{H}, \mathrm{CH}_{3}\right), 2.87\left(\mathrm{~s}, 3 \mathrm{H}, \mathrm{CH}_{3}\right)$, $1.54-1.51\left(\mathrm{t}, 3 \mathrm{H}, \mathrm{CH}_{3}(\mathrm{OEt})\right), 1.10-1.07\left(\mathrm{t}, 6 \mathrm{H}, \mathrm{CH}_{3}(\mathrm{EtOH})\right) .{ }^{13} \mathrm{C}$ NMR $\left(100 \mathrm{MHz}, \mathrm{DMSO}-d_{6}\right): \delta 171.89,171.39,166.55,166.22$, $166.03,159.23,158.05,150.01149 .87,134.68,133.78,132.85,132.59$, $131.98,131.72,130.73,130.61,129.93,129.56,129.33,127.99,127.56$, $126.52,126.16,124.95,124.33,119.51,118.45,118.16,117.99,117.89$ $116.40,115.38,115.13,111.26,110.04,80.15,56.57,24.19,23.96$, 19.03, 18.74. ${ }^{1} \mathrm{H}$ NMR (DMSO- $d_{6}+\mathrm{EtOH}, 400 \mathrm{MHz}$ ): $\delta 8.09-6.60$ (m, 10H, aromatic), 5.56-5.41 (m, 2H, $\left.\mathrm{CH}_{2}(\mathrm{OEt})\right), 4.51(\mathrm{~s}, 2 \mathrm{H}$, $\left.\mathrm{NH}_{2}\right), 3.52-3.47\left(\mathrm{q}, 48 \mathrm{H}, \mathrm{CH}_{2}(\mathrm{EtOH})\right), 2.86\left(\mathrm{~s}, 3 \mathrm{H}, \mathrm{CH}_{3}\right), 1.55-$ $1.52\left(\mathrm{t}, 3 \mathrm{H}, \mathrm{CH}_{3}(\mathrm{OEt})\right), 1.11-1.07\left(\mathrm{t}, 72 \mathrm{H}, \mathrm{CH}_{3}(\mathrm{EtOH})\right) .{ }^{13} \mathrm{C}$ NMR $\left(100 \mathrm{MHz}, \mathrm{DMSO}-d_{6}+\mathrm{EtOH}\right): \delta 171.97,165.94,158.21,149.79$, $133.63,132.36,131.91,130.51,129.14,127.34,126.02,124.18,119.28$, $118.09,116.28,115.27,111.23,80.00,56.61,23.64,18.50,18.35 .{ }^{51} \mathrm{~V}$ NMR (DMSO- $\left.d_{6} / \mathrm{MeCN}-d_{1}(1: 10)\right): \delta-511$ (100\%).

\section{RESULTS AND DISCUSSION}

Synthesis. The reaction of $\mathrm{NH}_{4} \mathrm{~V}^{\mathrm{V}} \mathrm{O}_{3}$ with an equimolar amount of $\mathrm{H}_{2}$ hnal-abhz ( $\left.\mathbf{L}^{1}\right), \mathrm{H}_{2}$ hnal-hbhz $\left(\mathbf{L}^{2}\right), \mathrm{H}_{2}$ han-bhz $\left(\mathbf{L}^{3}\right)$, and $\mathrm{H}_{2}$ han-abhz $\left(\mathbf{L}^{4}\right)$ (cf. Scheme 1 and eq 1 ) in EtOH yielded the oxidovanadium $(\mathrm{V})$ complexes $\left[\mathrm{V}^{\mathrm{V}} \mathrm{O}(\mathrm{OEt})(\mathrm{hnal}-\right.$ abhz) $]$ (1), [ $\left.\mathrm{V}^{\mathrm{V}} \mathrm{O}(\mathrm{OEt})(\mathrm{hnal}-\mathrm{hbhz})\right]$ (2), $\left[\mathrm{V}^{\mathrm{V}} \mathrm{O}(\mathrm{OEt})\right.$ (han$\mathrm{bhz})](3)$, and $\left[\mathrm{V}^{\mathrm{V}} \mathrm{O}(\mathrm{OEt})(\right.$ han-abhz $\left.)\right]$ (4), respectively, in good yield. These compounds are soluble in $\mathrm{CH}_{2} \mathrm{Cl}_{2}, \mathrm{~N}, \mathrm{~N}$ dimethylformamide, and DMSO, sparingly soluble in methanol, $\mathrm{EtOH}$, and $\mathrm{CH}_{3} \mathrm{CN}$, and diamagnetic and nonconducting in solution.

$$
\begin{aligned}
& \mathrm{NH}_{4} \mathrm{VO}_{3}+\mathrm{H}_{2} \mathrm{~L}+\mathrm{EtOH} \\
& \quad \rightarrow\left[\mathrm{V}^{\mathrm{V}} \mathrm{O}(\mathrm{OEt})(\mathrm{L})\right]+\mathrm{NH}_{4} \mathrm{OH}+\mathrm{H}_{2} \mathrm{O}
\end{aligned}
$$

Transformation of the structurally characterized $\left[\mathrm{V}^{\mathrm{V}} \mathrm{O}(\mathrm{OEt})\right.$ (L)] complexes in solution, leading to the formation of the corresponding monooxido-bridged divanadium $(\mathrm{V}, \mathrm{V})$ compounds $\left[\left\{\mathrm{V}^{\mathrm{V}} \mathrm{O}(\mathrm{L})\right\}_{2}-\mu\right.$-O $]$ (eq 2), is demonstrated. The mixed-valence vanadium(IV,V) species $\mathrm{V}_{2} \mathrm{O}_{3}{ }^{3+}$ were generated in solution by constant potential electroreduction, at $-0.10 \mathrm{~V}$, of the corresponding $\left[\left\{\mathrm{V}^{\mathrm{V}} \mathrm{O}(\mathrm{L})\right\}_{2}-\mu-\mathrm{O}\right]$ species (eq 3 ).

$$
\begin{aligned}
& 2\left[\mathrm{~V}^{\mathrm{V}} \mathrm{O}(\mathrm{OEt})(\mathrm{L})\right]+\mathrm{H}_{2} \mathrm{O} \leftrightarrows\left[\left\{\mathrm{V}^{\mathrm{V}} \mathrm{O}(\mathrm{L})\right\}_{2}-\mu-\mathrm{O}\right]+2 \mathrm{EtOH} \\
& {\left[\left\{\mathrm{V}^{\mathrm{V}} \mathrm{O}(\mathrm{L})\right\}_{2}-\mu-\mathrm{O}\right]+\mathrm{e}^{-} \rightarrow\left[\left\{\mathrm{V}^{\mathrm{V}} \mathrm{O}(\mathrm{L})\right\}\left\{\mathrm{V}^{\mathrm{IV}} \mathrm{O}(\mathrm{L})\right\}-\mu-\mathrm{O}\right]^{-}}
\end{aligned}
$$

Scheme 1 provides an overview of the compounds described in this work and of how they are obtained. The structural formulas of complexes are based on elemental analyses, spectroscopic (IR, UV-vis, EPR, ${ }^{1} \mathrm{H},{ }^{13} \mathrm{C}$, and ${ }^{51} \mathrm{~V} \mathrm{NMR}$, and ESI-MS) data, and single-crystal XRD analyses of compounds 1,3 , and 4 .

Structure Description. The IR spectra of complexes 1-4 showed the characteristic $\mathrm{V}=\mathrm{O}$ stretching band at ca. 991-999 $\mathrm{cm}^{-1}$, and the observed elemental $(\mathrm{C}, \mathrm{H}$, and $\mathrm{N}$ ) analytical data of all of them are consistent with their formulations as $\left[\mathrm{V}^{\mathrm{V}} \mathrm{O}(\mathrm{OEt})(\mathrm{L})\right]$ compounds. The preliminary characterization data (microanalysis and IR) indicated the presence of the ligand, oxido, and ethoxido moieties in the complexes. To confirm the binding mode of the azine-type ligands in these compounds, the molecular structure of some of the monomeric complexes was determined by a single-crystal XRD technique. The atom numbering for complexes $\mathbf{1}, \mathbf{3}$, and $\mathbf{4}$ is given in Figure 1 with the relevant bond distances and angles collected in Table 2. The ORTEP image of complex 4 identifies the C19 
Table 2. Selected Bond Distances ( $\AA$ ) and Angles (deg) for 1,3 , and 4

\begin{tabular}{llll} 
parameter & \multicolumn{1}{c}{$\mathbf{1}$} & \multicolumn{1}{c}{3} & \multicolumn{1}{c}{4} \\
$\mathrm{~V} 1-\mathrm{O} 1$ & $1.834(4)$ & $1.835(2)$ & $1.810(3)$ \\
$\mathrm{V} 1-\mathrm{O} 2$ & $1.914(4)$ & $1.908(2)$ & $1.897(3)$ \\
$\mathrm{V} 1-\mathrm{O} 3$ & $1.606(5)$ & $1.587(2)$ & $1.573(4)$ \\
$\mathrm{V} 1-\mathrm{O} 4$ & $1.730(7)$ & $1.753(2)$ & $1.712(5)$ \\
$\mathrm{V} 1-\mathrm{N} 1$ & $2.075(5)$ & $2.093(2)$ & $2.088(3)$ \\
$\mathrm{N} 1-\mathrm{N} 2$ & $1.384(6)$ & $1.395(3)$ & $1.396(5)$ \\
& & & \\
O1-V1-O3 & $106.70(2)$ & $108.11(8)$ & $107.40(2)$ \\
O1-V1-O4 & $99.40(3)$ & $99.03(8)$ & $96.90(2)$ \\
O1-V1-N1 & $82.50(2)$ & $81.91(7)$ & $82.10(1)$ \\
O2-V1-O3 & $107.10(2)$ & $110.68(9)$ & $106.90(2)$ \\
O2-V1-O4 & $90.20(3)$ & $89.39(8)$ & $89.20(2)$ \\
O2-V1-N1 & $74.40(2)$ & $74.36(7)$ & $75.10(1)$ \\
O3-V1-O4 & $105.10(3)$ & $105.20(9)$ & $109.50(2)$ \\
O3-V1-N1 & $97.00(2)$ & $97.15(8)$ & $97.40(2)$ \\
\hline
\end{tabular}

atom as slightly disordered, which is reflected as B-level CheckCIF alerts. In all compounds, the $\mathrm{V}^{\mathrm{V}}$ center is surrounded by a $\mathrm{O}_{4} \mathrm{~N}$ binding set, constituting a distorted square pyramidal structure, as indicated by the geometric parameter $\tau$ : 0.25 (1), 0.33 (3), and $0.19(4) .^{29}$ The basal plane is made up of the phenolato $\mathrm{O}$, enolato $\mathrm{O}$, and imine $\mathrm{N}$ atoms from the ligand and the $\mathrm{O}$ atom from deprotonated alkoxide. The Schiff base ligands form six- and five-membered chelate rings, with the $\mathrm{O} 1-\mathrm{V} 1-\mathrm{N} 1$ and $\mathrm{O} 2-\mathrm{V} 1-\mathrm{N} 1$ angles in the range of $82.50(2)-81.91(7)^{\circ}$ and $75.10(1)-74.36(7)^{\circ}$, respectively. The apical positions of the square pyramids are occupied by the terminal oxido $\mathrm{O} 3$ atoms. The short $\mathrm{V} 1-\mathrm{O} 3$ distance, in the range of $1.606(5)-1.573(4) \AA$, indicates the presence of a $\mathrm{V}-\mathrm{O}$ (oxido) bond, which is commonly found in five- and sixcoordinated octahedral complexes of vanadium(IV) and vanadium $(\mathrm{V}) .{ }^{15 \mathrm{~b}, 16}$ Considering compounds $\mathbf{1}, \mathbf{3}$, and $\mathbf{4}$, overall the $\mathrm{V}-\mathrm{O}$ bond lengths follow the order $\mathrm{V}-\mathrm{O}$ (oxido) $<\mathrm{V}-$ $\mathrm{O}$ (alkoxido) < $\mathrm{V}-\mathrm{O}$ (naphthalato) $<\mathrm{V}-\mathrm{O}$ (enolato). These data indicate stronger binding of the alkoxido moiety compared to those of naphthalato and enolato $\mathrm{O}$ atoms.

IR Spectra in Solution. The IR spectra of complexes 1-4 in chloroform/DMSO depict two $\mathrm{V}=\mathrm{O}$ stretching bands at $\sim 1004$ and $\sim 970 \mathrm{~cm}^{-1}$, which may be indicative of the presence of two distinct $\mathrm{V}=\mathrm{O}$ moieties in solution. ${ }^{12 \mathrm{~b}} \mathrm{~A}$ representative spectrum is shown in Figure S1. Another new band observed in solution at around $820-823 \mathrm{~cm}^{-1}$ was tentatively assigned to the $\nu(\mathrm{V}-\mathrm{O}-\mathrm{V})^{12 \mathrm{~b}, 17 \mathrm{f}, 18 \mathrm{~b}, 24 \mathrm{c}, 30}$ mode, which was not observed in the spectra of 1-4 in the solid state. These observations indicate that at least two distinct species may exist in solution. The IR spectra in solution depict many features already observed in the solid state, corresponding to the mononuclear complexes, as well as features that probably correspond to the simultaneous formation of the corresponding dinuclear complexes as second species.

Electronic Spectroscopy. The electronic spectral data of 1-4 are summarized in the Experimental Section, and a representative spectrum is shown in Figure S2. Each displays four strong absorption bands in $\mathrm{CHCl}_{3}$ in the wavelength range 435-235 $\mathrm{nm}$. The lower-energy absorptions in the range 435$420 \mathrm{~nm}$ are ascribed to the ligand-to-metal charge-transfer transitions, while the higher-energy absorptions in the range $340-235 \mathrm{~nm}$ are likely to be due to ligand-centered transitions. ${ }^{12 \mathrm{~b}, 30}$ The electronic spectra depict similar features but do not allow clear conclusions regarding the possibility of the existence of mononuclear- dinuclear equilibria.

NMR Spectral Studies. The ${ }^{1} \mathrm{H}$ NMR spectra of $\mathbf{1 - 4}$ in DMSO- $d_{6}$ (which contained trace water) yielded two close but separate sets of bands in an approximate 1:1 ratio (part a, Figure $2 \mathrm{~A}, \mathrm{~B} ;{ }^{1} \mathrm{H}$ NMR spectra of $\mathbf{1}$ are taken as representative). The set of sharp ${ }^{1} \mathrm{H}$ NMR signals marked with pink rectangles correspond to the monomeric form $\mathbf{M}^{\mathrm{V}}$ of species $\mathbf{1}$, which was isolated in the solid state and structurally characterized. The relatively less sharp signals marked with blue rectangles were assigned to a distinct species designated by $\mathbf{M}^{\mathrm{V}}-\mathbf{M}^{\mathbf{V}}$ (the corresponding second species, probably a dinuclear $\mu$-oxido complex) generated in situ in solution. So, as is also discussed below, the ${ }^{1} \mathrm{H}$ NMR results for $\mathbf{1 - 4}$ indicate that, in each case, at least two species exist in DMSO solution.

The spectra of 1 and 2 exhibit two sets of $-\mathrm{CH}$ singlets in the ranges of 9.99-9.94 and 10.09-9.95 ppm, respectively. The spectrum of 2 exhibits two sets of $-\mathrm{OH}$ (phenolic) resonances at 11.60 and $11.42 \mathrm{ppm}$. The spectra of 3 and 4 exhibit two sets of $-\mathrm{CH}_{3}$ (methyl) resonances in the ranges of 2.92-2.84 and 2.95-2.87 ppm, respectively. The spectra of 1 and 4 exhibit a broad signal for two $-\mathrm{NH}_{2}$ protons in the range of $4.52-4.32$ ppm. Finally, the aromatic protons of $\mathbf{1}, \mathbf{2}$, and $\mathbf{4}$ (a total of 20) and of 3 (a total of 22) appear in the range of 8.65-6.31 ppm. In addition, all of the complexes exhibit separate bands corresponding to bound ethoxido in the ranges of 5.86-5.41 ppm $\left(-\mathrm{CH}_{2}\right)$ and $1.63-1.48 \mathrm{ppm}\left(-\mathrm{CH}_{3}\right),{ }^{12 \mathrm{~b}, \mathrm{~d}, 17 \mathrm{q}}$ and the sharp peaks observed in the ranges of $3.50-3.41 \mathrm{ppm}\left(-\mathrm{CH}_{2}\right)$ and $1.10-1.04 \mathrm{ppm}\left(-\mathrm{CH}_{3}\right)$ were assigned to free, nonligated $\mathrm{EtOH}$ that is most likely to be formed in situ in solution. Although the chemical shifts of the methylene and methyl protons of $\mathrm{EtOH}$ are very close to those of free $\mathrm{EtOH}$, the alcoholic proton is not detected, probably because of the low concentration or overlap of signals.

Upon the addition of a few drops of EtOH to the DMSO solution of 1-4, the second set of bands (blue) disappeared and cleaner spectra were obtained (part b, Figure 2A,B); these exhibit only one $-\mathrm{OH}$ (phenolic) resonance at $11.61 \mathrm{ppm}$ for 2 , one $-\mathrm{CH}$ proton signal in the range of 9.94-9.80 ppm for $\mathbf{1}$ and 2, and signals for a total of 10 aromatic protons for $\mathbf{1}, \mathbf{2}$, and 4 and 11 aromatic protons for 3 in the range of 8.56-6.57 ppm. In addition, the coordinated ethoxido bands in the ranges of $5.86-5.41 \mathrm{ppm}\left(-\mathrm{CH}_{2}\right)$ and $1.63-1.49 \mathrm{ppm}$ $\left(-\mathrm{CH}_{3}\right)^{12 \mathrm{~b}, \mathrm{~d}, 17 \mathrm{q}}$ broadened, and the intensity of the free $\mathrm{EtOH}$ bands in the ranges of 3.52-3.41 ppm $\left(-\mathrm{CH}_{2}\right)$ and 1.11-1.04 ppm $\left(-\mathrm{CH}_{3}\right)$ increased.

The ${ }^{13} \mathrm{C}$ NMR spectra of $\mathbf{1 - 4}$ in DMSO also showed two sets of bands with an approximate ratio of 1:1; the second set of bands (blue) disappeared upon the addition of a small amount of EtOH, as in the case of the ${ }^{1} \mathrm{H}$ NMR spectrum. The representative ${ }^{13} \mathrm{C}$ NMR spectra of $\mathbf{1}$ are included in Figure S3.

Further characterization of the vanadium $(\mathrm{V})$ complexes was obtained by measuring their ${ }^{51} \mathrm{~V}$ NMR spectra in solution; namely, the ${ }^{51} \mathrm{~V}$ NMR chemical shift values $\delta\left({ }^{51} \mathrm{~V}\right)$ of the compounds recorded in MeCN-DMSO (10:1) were obtained to access their behavior/speciation upon modification of the composition of the solutions (Scheme 1 and Table 3). The assignment of the resonances was done by considering both the experiments described below and the results of the DFT calculations carried out; the latter also demonstrate a weak dependence of the ${ }^{51} \mathrm{~V}$ chemical shift on the solvent nature. The ${ }^{51} \mathrm{~V}$ NMR spectral data of these complexes show strong resonances at $\delta-548(1), \delta-554(2), \delta-500(3)$, and $\delta-511$ 
(A)
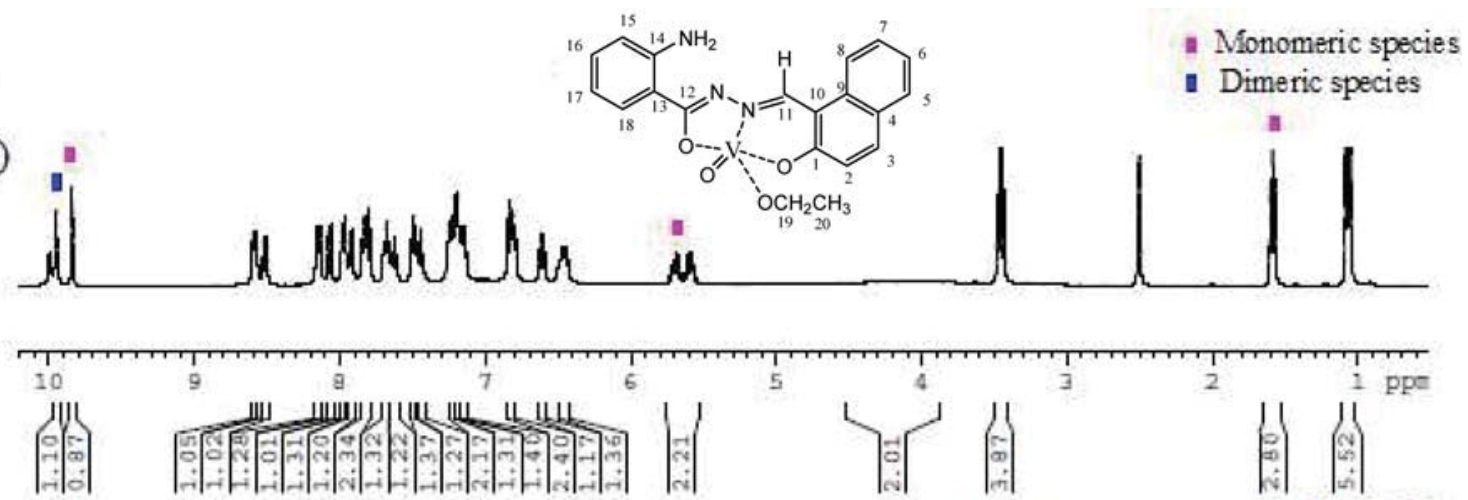

b)

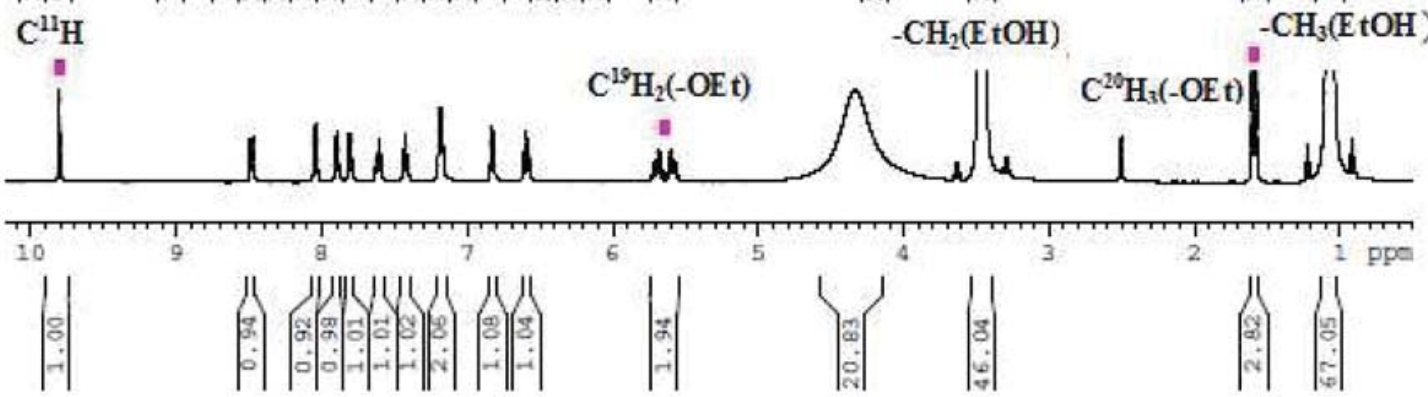

(B)

a)

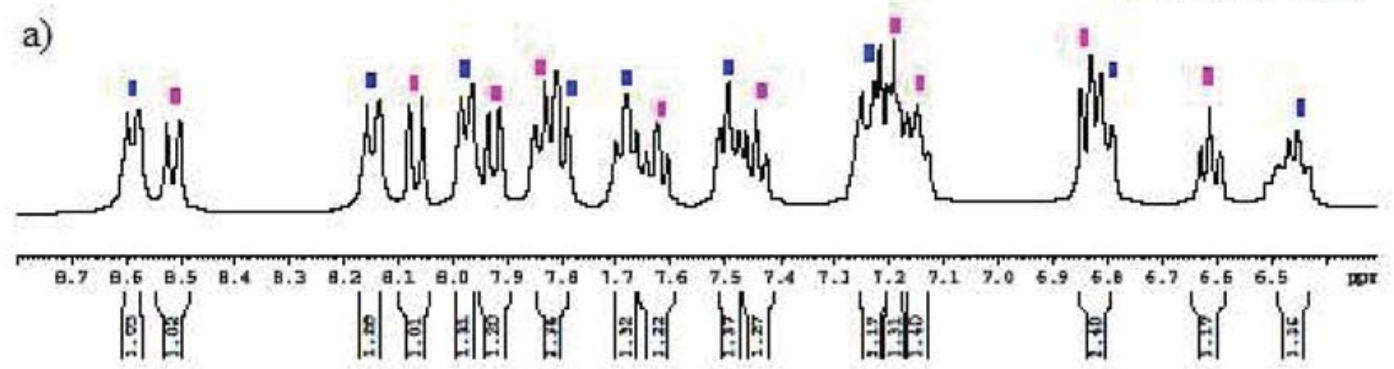

b)

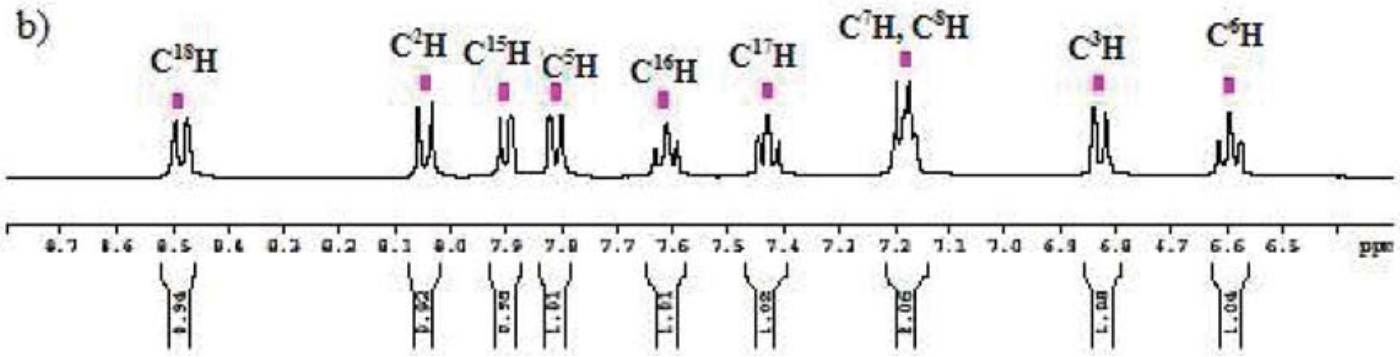

Figure 2. ${ }^{1} \mathrm{H}$ NMR spectra of complex 1 in DMSO-d $d_{6}$ (A) full range; (B) aromatic region; (a) before the addition of EtOH; (b) after the addition of EtOH.

ppm (4) in DMSO- $d_{6}: \mathrm{MeCN}-d_{1}(1: 10)$, values within those normally found for vanadium $(\mathrm{V})$ complexes containing $\mathrm{O} / \mathrm{N}$ donor sets. ${ }^{1,8,31,32}$

Table 3 summarizes the data of experimental and calculated ${ }^{51} \mathrm{~V}$ NMR chemical shifts obtained. Namely, it depicts data concerning the solvent-dependent behavior of the ${ }^{51} \mathrm{~V}$ NMR spectra. If coordination of a solvent molecule (DMSO, $\mathrm{MeCN}$, or EtOH) to vanadium in the monomeric complexes $\mathbf{1 - 4}$ is considered, theoretical DFT calculations indicate that the formation of $\mathbf{1} \cdot \mathrm{DMSO}\left(\delta_{\mathrm{V}}{ }^{\text {calc }}=-570 \mathrm{ppm}\right), \mathbf{1} \cdot \mathrm{MeCN}\left(\delta_{\mathrm{V}}{ }^{\text {calc }}=\right.$ $-579 \mathrm{ppm})$, and $\mathbf{1} \cdot \mathrm{EtOH}\left(\delta_{\mathrm{V}}{ }^{\text {calc }}=-587 \mathrm{ppm}\right)$ adducts from 1 and the corresponding solvent is significantly endergonic (by $13.1,16.0$, and $13.9 \mathrm{kcal} / \mathrm{mol}$, respectively, in terms of the Gibbs free energy in solution). Thus, we do not expect the formation of these species to be favorable. Cartesian atomic coordinates (in $\AA$ ) of the calculated equilibrium structures, for DMSO taken as the solvent, for the calculations of the bulky solvent effect are summarized in Table S1. 
Table 3. Summary of the Experimental ${ }^{51} \mathrm{~V}$ NMR Data $\left(\delta_{\mathrm{V}}\right.$, ppm) and DFT Calculations $\left(\delta_{\mathrm{V}}{ }^{\text {calc }}, \mathrm{ppm}\right.$, in parentheses, for DMSO solutions if not Stated Otherwise): See the Text and Scheme 1

\begin{tabular}{|c|c|c|}
\hline compound & $\mathbf{M}^{\mathrm{V}}$ & $\begin{array}{l}\mathbf{M}^{\mathrm{V}}-\mathbf{M}^{\mathrm{V}}(\text { dinuclear } \\
\text { species) }\end{array}$ \\
\hline 1 & $\begin{array}{l}-548(-566),(-568),{ }^{a}(-568),{ }^{b} \\
\quad(-566)^{c}\end{array}$ & $-576(-587,-573)$ \\
\hline $1 \cdot \mathrm{DMSO}$ & $(-570)$ & \\
\hline $1 \cdot \mathrm{EtOH}$ & $(-587)$ & \\
\hline $1 \cdot \mathrm{MeCN}$ & $(-579)$ & \\
\hline 2 & $-554(-553)$ & -594 \\
\hline 3 & $-500(-529)$ & -522 \\
\hline 4 & $-511(-539),(-539)^{c}$ & $-549(-566)$ \\
\hline $4 \cdot \mathrm{DMSO}$ & $(-543)$ & \\
\hline $4 \cdot \mathrm{EtOH}$ & $(-561)$ & \\
\hline
\end{tabular}

${ }^{a}$ Geometry optimization with DMSO as the solvent, and single-point NMR calculations with $\mathrm{MeCN}$ as the solvent. ${ }^{b}$ Geometry optimization with DMSO as the solvent, and single-point NMR calculations with $\mathrm{EtOH}$ as the solvent. ${ }^{c}$ Both geometry optimization and NMR calculations with $\mathrm{EtOH}$ as the solvent.

The ${ }^{51} \mathrm{~V}$ NMR spectrum of 1 (ca. $4.0 \mathrm{mM}$ ) dissolved in $\mathrm{MeCN}-\mathrm{DMSO}$ has a major resonance at $-548 \mathrm{ppm}$, a minor one at $-576 \mathrm{ppm}$, and one/two other very minor peaks [Figure $3 \mathrm{~A}(\mathrm{a})$ ]. Complex 1 (Scheme 1) probably corresponds to the
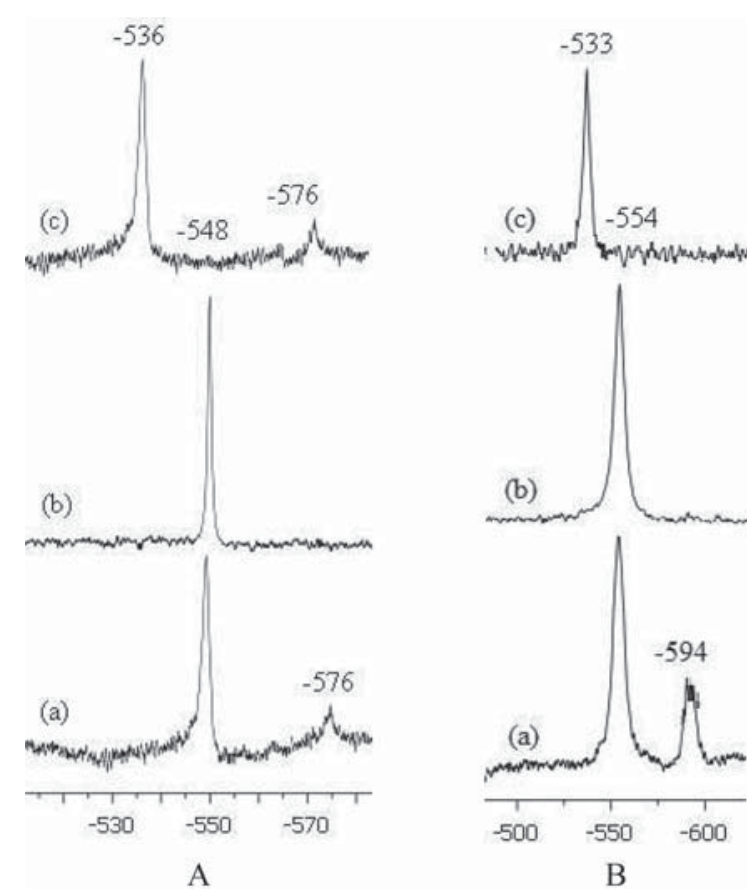

Figure 3. ${ }^{51} \mathrm{~V}$ NMR spectra of $4.0 \mathrm{mM}$ solutions of (A) $\mathbf{1}$ and (B) $\mathbf{2}$ : (a) in MeCN:DMSO (10:1); (b) solutions of part a after the addition of a few drops of EtOH; (c) solutions of part b after $6 \mathrm{~h}$.

resonance at $-548 \mathrm{ppm}$; this agrees reasonably with a $\delta_{\mathrm{V}}{ }^{\text {calc }}$ value of $-566 \mathrm{ppm}$ (Table 3 ). The minor species observed in Figure $3 \mathrm{~A}(\mathrm{a})$ at $-576 \mathrm{ppm}$ may tentatively be assigned to $\mathbf{1}^{\mathrm{V}}$ $\mathbf{1}^{\mathrm{V}} \cdot \mathrm{DMSO}$ and $\mathbf{1}^{\mathrm{V}}-\mathbf{1}^{\mathrm{V}} \cdot \mathrm{MeCN}$; in fact, the dinuclear complex $\mathbf{1}^{\mathrm{V}}$ $\mathbf{1}^{\mathrm{V}}$ (Scheme 1) corresponds to calculated $\delta_{\mathrm{V}}{ }^{\text {calc }}$ values of -587 and -573 ppm.

Upon the addition of a few drops of EtOH, all minor peaks in Figure $3 \mathrm{~A}(\mathrm{a})$ disappear and only the one at $\delta_{\mathrm{V}}=-548 \mathrm{ppm}$ is observed [Figure $3 \mathrm{~A}(\mathrm{~b})]$. After ca. $6 \mathrm{~h}$, a clear major species shows up at $-536 \mathrm{ppm}$, and the resonance at $-576 \mathrm{ppm}$ is again detected. Probably most $\mathrm{V}^{\mathrm{V}}$-hnal-abhz complexes decomposed with time upon the addition of $\mathrm{EtOH}$, with the resonance at $-536 \mathrm{ppm}$ being compatible with those reported for vanadate ethanol esters $\left(\mathrm{ROVO}_{3}{ }^{2-}\right)$ or vanadates, ${ }^{31}$ whose $\delta_{\mathrm{V}}$ may vary with the $\mathrm{pH}^{33}$ and probably also slightly with the nature of the solvent.

Complex 2 in $\mathrm{MeCN}-\mathrm{DMSO}$ depicts a resonance at -554 ppm [Figure $3 \mathrm{~B}(\mathrm{a})]$. The corresponding $\delta_{\mathrm{V}}{ }^{\text {calc }}=-553 \mathrm{ppm}$ supports its assignment to 2 . The resonance measured at $\delta_{\mathrm{V}}=$ $-594 \mathrm{ppm}$ is tentatively assigned to the corresponding species $2^{\mathrm{V}}-2^{\mathrm{V}}$ (Scheme 1). The signal at $\delta_{\mathrm{V}}=-594 \mathrm{ppm}$ almost disappears upon the addition of $\mathrm{EtOH}$ [Figure $3 \mathrm{~B}(\mathrm{~b})$ ], and after $6 \mathrm{~h}$, only a resonance at $-533 \mathrm{ppm}$ is detected. Again we tentatively assign this resonance to vanadates or vanadate ethanol esters $\left(\mathrm{ROVO}_{3}{ }^{2-}\right) \cdot{ }^{31,33}$ The ${ }^{51} \mathrm{~V}$ NMR spectrum of 3 dissolved in MeCN-DMSO depicts a single resonance at -500 ppm (Figure 4A), which we assign to 3 (Scheme 1); for this
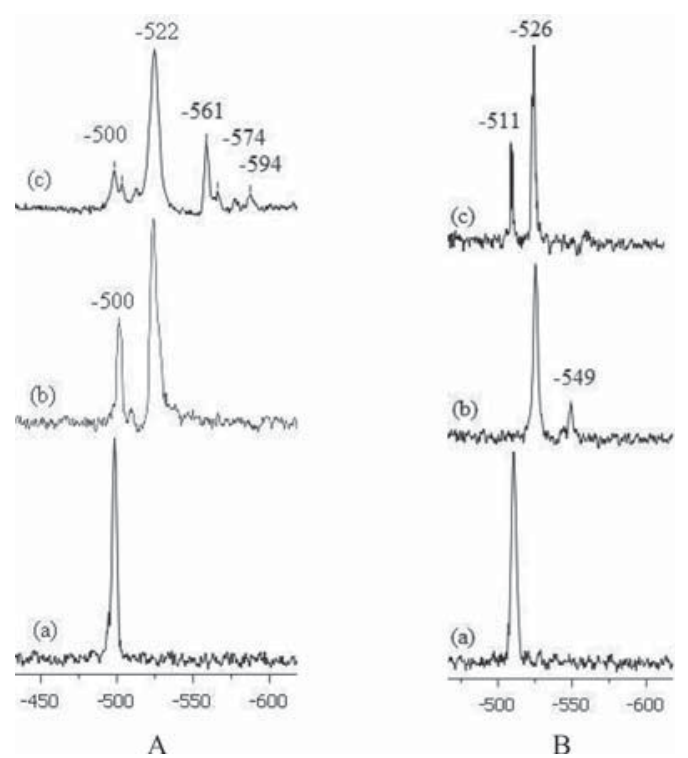

Figure 4. ${ }^{51} \mathrm{~V}$ NMR spectra of a $4.0 \mathrm{mM}$ solutions of (A) 3 and (B) 4 : (a) in MeCN:DMSO (10:1); (b) solutions of part a after $6 \mathrm{~h}$; (c) solutions of part $b$ after $12 \mathrm{~h}$.

species, $\delta_{\mathrm{V}}^{\text {calc }}=-529 \mathrm{ppm}$. After ca. $6 \mathrm{~h}$, this peak decreases in intensity and a major resonance appears at $-522 \mathrm{ppm}$, which we tentatively assign to the corresponding $\left[\left\{\mathrm{V}^{\mathrm{V}} \mathrm{O}\left(\mathbf{L}^{3}\right)\right\}_{2}-\mu\right.$-O $]$ (species $3^{\mathrm{V}}-3^{\mathrm{V}}$; Scheme 1). After $12 \mathrm{~h}$, several other minor peaks are detected; these may correspond to vanadate ethanol esters $\left(\mathrm{ROVO}_{3}{ }^{2-}\right.$ and $\left.\mathrm{ROVO}_{3} \mathrm{H}^{-}\right)$and/or to vanadates $\left(\mathrm{V}_{1}, \mathrm{~V}_{2}\right.$, or $\left.\mathrm{V}_{4}\right)^{31,33}$

In the case of 4 dissolved in MeCN-DMSO, it depicts a resonance at $\delta_{\mathrm{V}}=-511 \mathrm{ppm}$ (Figure 4B), which may be assigned to 4 (Scheme 1); for this species in DMSO, $\delta_{\mathrm{V}}^{\text {calc }}=$ $-539 \mathrm{ppm}$. After ca. $6 \mathrm{~h}$, this peak disappears, and two other resonances are detected: a major one at $-526 \mathrm{ppm}$ and a minor one at $-549 \mathrm{ppm}$. We tentatively assign the resonance at -526 $\mathrm{ppm}$ to the corresponding $\left[\left\{\mathrm{V}^{\mathrm{V}} \mathrm{O}\left(\mathrm{L}^{4}\right)\right\}_{2}-\mu-\mathrm{O}\right]$ (species $4^{\mathrm{V}}-\mathbf{4}^{\mathrm{V}}$; Scheme 1) and the one at $-549 \mathrm{ppm}$ to vanadates or vanadate ethanol esters $\left(\mathrm{ROVO}_{3}{ }^{2-}\right) .{ }^{31,33}$ After $12 \mathrm{~h}$, the latter resonance disappears.

In agreement with the IR in solution, the NMR $\left({ }^{1} \mathrm{H},{ }^{13} \mathrm{C}\right.$, and ${ }^{51} \mathrm{~V}$ ) data also indicate that at least two species exist in a 
DMSO/MeCN-DMSO solution (either immediately or after a few hours), and whatever the nature of the second species (here identified as $\mathbf{M}^{\mathrm{V}}-\mathbf{M}^{\mathrm{V}}$ ), it converts back to its monomeric form $\mathbf{M}^{\mathrm{V}}$ in the presence of a small amount of EtOH.

Alkoxides often form dinuclear complexes, so one of the possible structures for the dinuclear species is the formation of an alkoxido-bridged dimer. ${ }^{34}$ However, we could exclude the possibility of an ethoxido-bridged dinuclear complex as the second species because there is only one set of bands for a coordinated ethoxido moiety. If the second species is an ethoxido-bridged dimer, there should be another set of bands for two bridging ethoxido groups, and as a six-coordinated complex, the $\mathrm{V}=\mathrm{O}$ stretching should have been found at lower frequency. ${ }^{12 c}$ Additionally, theoretical DFT calculations indicate that the Gibbs free energy of formation of such dinuclear species 4-4a from two monomers of 4 (taken as representative, Scheme 2) is highly endergonic in both

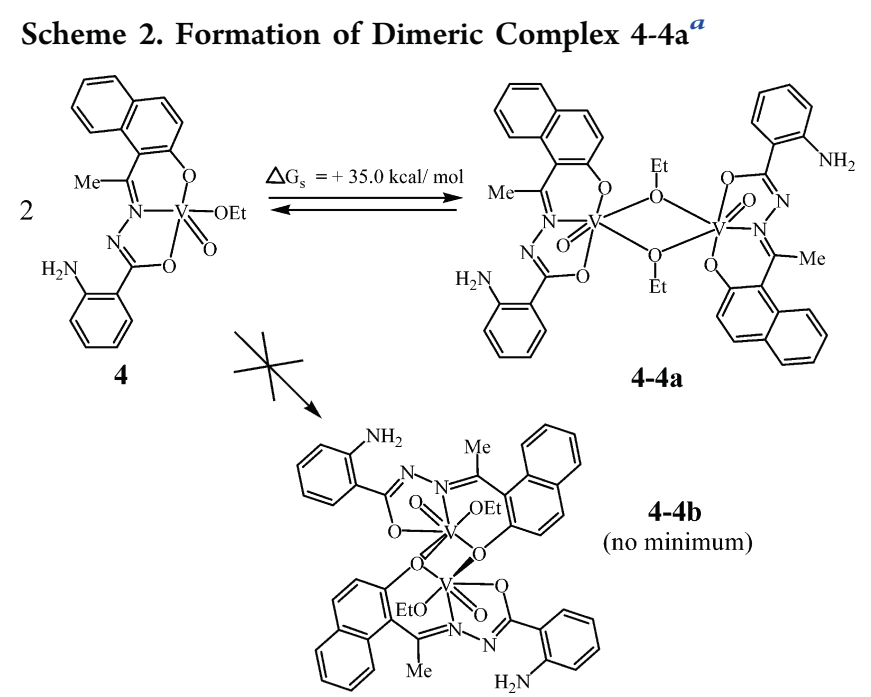

${ }^{a}$ The calculated Gibbs free energy of the reaction in DMSO solution is indicated. The molecular formula of $\mathbf{4 - 4 b}$, for which no minimum was found in the calculation, is also shown (see the text).

DMSO and EtOH solutions $\left(\Delta G_{\mathrm{s}}\right.$ values are +35.0 and +32.4 $\mathrm{kcal} / \mathrm{mol}$, respectively). So, we may exclude the possibility of such alkoxido-bridged dimers as the second species.

Other possible structures for second species are a chain structure in which a $\mathrm{O}$ phenolate atom of the neighboring monomeric species would link $\mathrm{V}$ centers of the monomeric complexes. We also exclude the possibility of such a phenolatelinked chain structure; if phenolate was coordinated to the $\mathrm{V}$ center in the minor species, a phenolate ligand to vanadium metal charge-transfer band would have disappeared when a small amount of EtOH was added to the chloroform solution of complexes 1-4. However, there were no such recognizable changes in the UV-vis spectra of complexes 1-4 in the chloroform solution after the addition of a small amount of EtOH. Additionally, theoretical DFT calculations demonstrate that such a dinuclear species $\mathbf{4} \mathbf{- 4 b}$ probably does not exist in solution because all attempts to locate the corresponding minimum on the potential energy surface resulted in the formation of two separate monomers of 4 .

Finally, another of the plausible structures for the second species formed in solution is a dinuclear $\mu$-oxido complex. From the IR results in solution, there was a significant change in the $\mathrm{V}=\mathrm{O}$ stretches of complexes 1-4 in a chloroform/ DMSO solution compared to that of the monomeric species in the solid state, and another new band that may correspond to the $\nu(\mathrm{V}-\mathrm{O}-\mathrm{V})^{12 \mathrm{~b}, 30}$ mode is observed. This indicates that, along with monomeric species, the complexes may exist as an isolated dinuclear $\mu$-oxido (second species) in solution, as reported in other recent publications. ${ }^{12 f, 30}$

On the basis of the above results, the mentioned solution behavior of 1-4 should be ascribed to the equilibria in Scheme 3 existing in chloroform/DMSO/MeCN-DMSO containing a small amount of water, which also explains the simultaneous presence of the two species $\mathbf{M}^{\mathrm{V}}$ (monomer, isolated in the solid state and characterized by single-crystal XRD) and $\mathbf{M}^{\mathrm{V}}-\mathbf{M}^{\mathbf{V}}$ (second species, proposed as a dinuclear $\mu$-oxido complex) in solution.

Even though no water molecules were observed in the crystal structures of $\mathbf{1 - 4}$, possible proton sources may come from solvents used in the IR and NMR experiments or from water molecules in air that were probably adsorbed on the surface of the particles of the complexes. The role of water as well as of the free EtOH molecules for the existence of the above equilibria was inferred from the presence of the band at $\sim 3.73$ (br) ppm for water and the sharp peaks at $\sim 3.47$ and $\sim 1.07$ ppm from the protons of free, nonligated EtOH in DMSO- $d_{6}$. In some cases (2 and 3 ), the water peak is assigned, and in other cases (1 and 4), it is not clearly detected. However, these equilibria could be shifted more to the left by the addition of a few drops of EtOH, which was reflected in the change in the

Scheme 3. Schematic Diagram for the Interconversion of Monomeric and Dinuclear Species in Solution, Taking Complex 4 as Representative $^{a}$<smiles>CCOP1(=O)OC(c2ccccc2N)=NN1C(C)=O</smiles>

Monomeric form $\left(4^{\mathbf{v}}\right)$, isolated in the solid state

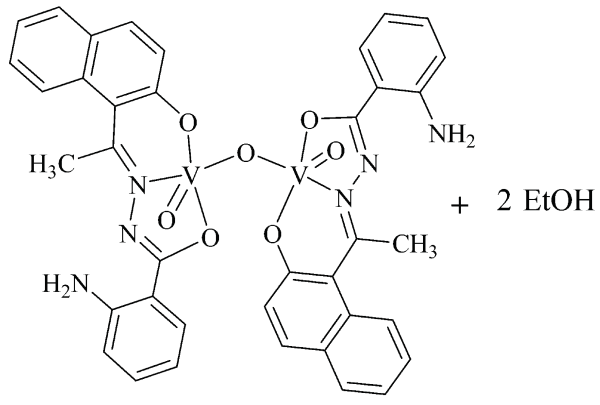

Dinuclear form $\left(4^{\mathrm{v}}-4^{\mathrm{v}}\right), 2^{\text {nd }}$ species in solution

${ }^{a}$ The $\Delta G_{s}$ values calculated using different functionals are indicated in kilocalories per mole. 
NMR spectrum of $\left[\mathrm{V}^{\mathrm{V}} \mathrm{O}(\mathrm{OEt})\left(\mathrm{L}^{1-4}\right)\right]$ upon the addition of EtOH.

These conclusions are supported by DFT calculations, which indicate that the Gibbs free energy of formation of a dinuclear complex of 4 shown in Scheme 3 is only $+2-3 \mathrm{kcal} / \mathrm{mol}$ in a DMSO solution. Such low positive values suggest that (i) the dinuclear complex may be easily formed in a DMSO solution in a noticeable amount, (ii) the monomer is still the predominant form in solution, in agreement with the higher intensity of its signal in the ${ }^{51} \mathrm{~V}$ NMR spectra compared to that of the dinuclear complex, and (iii) the addition of even a small amount of EtOH should efficiently shift this equilibrium toward monomeric structures, again in agreement with the experiment.

Therefore, we can conclude that, in a DMSO/MeCN-DMSO solution in the presence of $\mathrm{EtOH}$, compounds 1-4 partly retain the structure in which they are present in the solid state, and the other second species, $\mathbf{M}^{\mathrm{V}}-\mathbf{M}^{\mathrm{V}}$, which were also present in solution, may be reconverted into the mononuclear one, $\mathbf{M}^{\mathrm{V}}$. Complexes $\mathbf{M}^{\mathrm{V}}-\mathbf{M}^{\mathrm{V}}$ exhibit the characteristic spectroscopic properties of the dinuclear $\mu$-oxido species, similar to those of the dinuclear $\mu$-oxido complex reported in two of our recent publications. ${ }^{12 f, 30}$

ESI-MS. Complexes 1-4 in a DMSO solution gave two peaks at $m / z \sim 400-385$ and $\sim 784-754$ in the ESI-MS (negative mode) spectrum. The lower mass peak corresponds to the mononuclear complex anion, $\left[\mathrm{V}^{\mathrm{V}} \mathrm{O}_{2} \mathrm{~L}\right]^{-}$, isolated in the solid state, and for the other peak, we propose it corresponds to the molecular anion $\left[\mathrm{V}^{\mathrm{V}} \mathrm{V}^{\mathrm{IV}} \mathrm{O}_{3}(\mathrm{~L})_{2}\right]^{-}$of their dinuclear $\mu$-oxido species formed in solution. The representative ESI-MS spectrum for $\mathbf{2}$ is depicted in Figure 5. For complexes 1, 3,

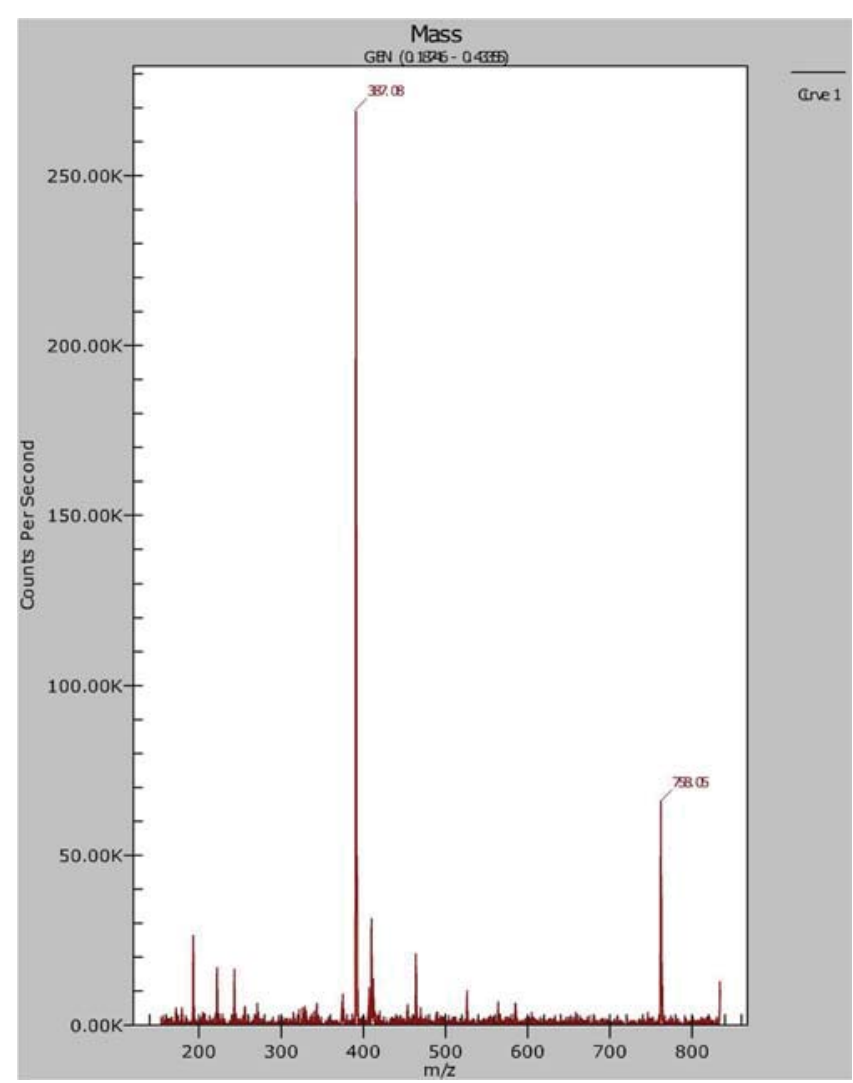

Figure 5. ESI-MS spectrum of complex 2 in DMSO: $\mathrm{m} / z 387.08$ $\left(100 \%,\left[\mathrm{~V}^{\mathrm{V}} \mathrm{O}_{2}\left(\mathbf{L}^{2}\right)\right]^{-}, \quad \mathrm{MW}=387.02\right)$ and $758.05(30 \%$, $\left[\mathrm{V}^{\mathrm{V}} \mathrm{V}^{\mathrm{IV}} \mathrm{O}_{3}\left(\mathrm{~L}^{2}\right)_{2}\right]^{-}$, $\left.\mathrm{MW}=758.48\right)$. and 4, rather similar MS results were obtained (Figures S4S6). For 1, 2, and 4, the peak corresponding to the mass of the mononuclear ion is predominant $(100 \%)$, whereas for 3 , it is the opposite: the peak corresponding to the mass of the dinuclear $\mu$-oxido ion is the predominant one. This difference in the behavior of $\mathbf{3}$ may be attributed to the absence of any $\mathrm{H}$ bond-acceptor atom as the second substituent on the phenyl ring of the coordinated ligand, which diminishes the possibility of any intramolecular hydrogen bonding. The results of mass spectrometry give strong evidence in favor of the formation of $\mu$-oxidodioxidodivanadium complexes as the second species in all systems.

Redox Properties. The redox properties of compounds $\left[\mathrm{V}^{\mathrm{V}} \mathrm{O}(\mathrm{OEt})(\mathrm{L})\right](\mathbf{1}-\mathbf{4})$ were studied by $\mathrm{CV}$ in $\mathrm{MeCN}$ using $\left[\mathrm{NBu}_{4}\right]\left[\mathrm{BF}_{4}\right]$ as the electrolyte. The cyclic voltammograms of the complexes display quasi-reversible cathodic waves in the range of $0.27-0.44 \mathrm{~V}$ (vs SCE), assigned to the $\mathrm{V}^{\mathrm{V}} \rightarrow \mathrm{V}^{\mathrm{IV}}$ reduction processes (Figure $\mathrm{S} 7$ ).

When compound $\mathbf{2}$ is left in the electrolyte solution for about $1 \mathrm{~h}$, a shift in the potential to lower values (by ca. $0.11 \mathrm{~V}$ ) of the cathodic wave is observed (Figure 6). The potential of the new

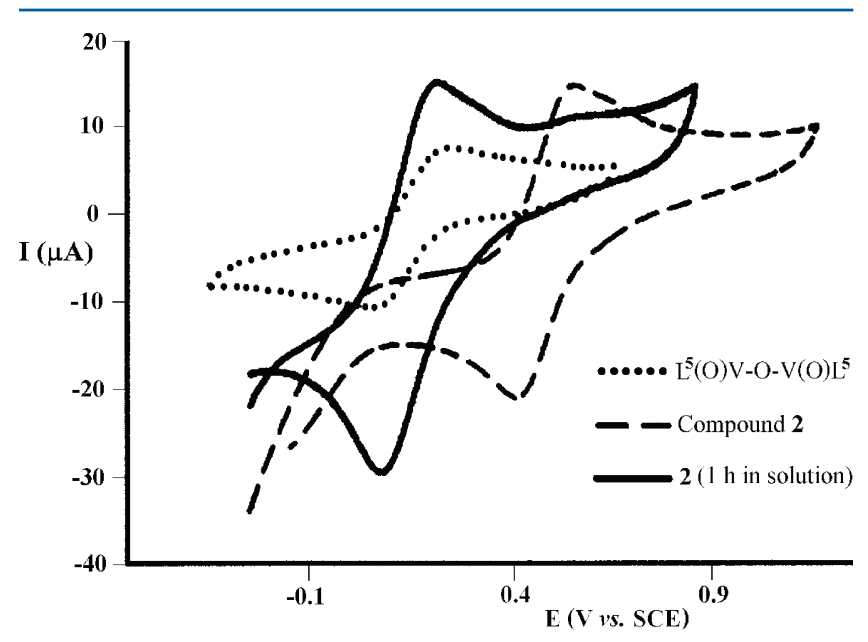

Figure 6. Cyclic voltammograms of 2 (---) immediately after dissolution and (-) after $1 \mathrm{~h}$ in the electrolyte solution and $(\bullet \bullet \bullet)$ of $\left[\left(\mathrm{V}^{\mathrm{V}} \mathrm{OL}^{5}\right)_{2} \mathrm{O}\right]\left(\mathrm{H}_{2} \mathrm{~L}^{5}=2\right.$-furoylazine of 2-hydroxy-1-acetonaphthone $)^{30}$ in $\left[\mathrm{NBu}_{4}\right]\left[\mathrm{BF}_{4}\right] / \mathrm{MeCN}(0.10 \mathrm{M})$ solutions.

wave is well in the range of that of the dinuclear $\mu$ oxidovanadium $(\mathrm{V})$ compound $\left[\mathbf{L}^{5}(\mathrm{O}) \mathrm{V}^{\mathrm{V}} \mathrm{OV}^{\mathrm{V}}(\mathrm{O}) \mathbf{L}^{5}\right]$ previously reported for a complex with a similar ligand $\left(\mathrm{L}^{5}\right.$, where $\mathrm{H}_{2} \mathbf{L}^{\mathbf{5}}=2$-furoylazine of 2-hydroxy-1-acetonaphthone). ${ }^{30} \mathrm{In}$ Figure 6, for comparative purposes, the cyclic voltammogram of $\left[\left(\mathrm{V}^{\mathrm{V}} \mathrm{OL}^{5}\right)_{2} \mathrm{O}\right]$ is also included; the similarity of the cyclic voltammograms of $\left[\mathbf{L}^{5}(\mathrm{O}) \mathrm{V}^{\mathrm{V}} \mathrm{OV}^{\mathrm{V}}(\mathrm{O}) \mathbf{L}^{5}\right]$ and $\mathbf{2}$ (after standing ca. $1 \mathrm{~h}$ in the electrolyte solution) further confirms the feasibility of the formation of $2^{\mathrm{V}}-2^{\mathrm{V}}$ in solutions of $\mathbf{2}$.

Chronocoulometry of 1-4 and Subsequent EPR Studies. In $\mathrm{MeCN}$, compounds 1-4 partly convert into the monooxido-bridged divanadium $(\mathrm{V}, \mathrm{V})$ complexes $\mathbf{M}^{\mathrm{V}}-\mathrm{M}^{\mathrm{V}}$, which most probably correspond to the precursors for monooxido-bridged divanadium(IV,V) compounds.

With the purpose of elucidating whether the mixed-valence species $\left[\left\{\mathrm{V}^{\mathrm{V}} \mathrm{V}^{\mathrm{IV}} \mathrm{O}_{2}(\mathrm{~L})_{2}\right\}-\mu\right.$-O $]$ (designated as $\mathbf{M}^{\mathrm{V}}-\mathbf{M}^{\mathrm{IV}}$, where $\mathbf{M}$ $=1,2,3$, or 4) could be obtained from complexes 1-4, CPE was undertaken at potentials selected according to the values measured by CV for the reduction of each complex (Table 4). 
Table 4. Spin Hamiltonian Parameters Obtained from 4.0 mM Solutions of the Reduced Forms of Compounds 1-4 in $\mathrm{MeCN}$ and Those Calculated by Simulation of the Experimental First-Derivative EPR Spectra Recorded at $77 \mathrm{~K}$ and DFT-Calculated Values for the Dinuclear Species $\left[\left\{\mathrm{VO}\left(\mathrm{L}^{1-4}\right)\right\}_{2}-\mu-\mathrm{O}\right]^{-}\left(1^{\mathrm{V}}-1^{\mathrm{IV}}, 2^{\mathrm{V}}-2^{\mathrm{IV}}, 3^{\mathrm{V}}-3^{\mathrm{IV}}\right.$, and $\left.4^{\mathrm{V}}-4^{\mathrm{IV}}\right)$, Monomeric $\left[\mathrm{V}^{\mathrm{IV}} \mathrm{O}(\mathrm{OEt})\left(\mathrm{L}^{1-4}\right)\right]^{-}$(Designated by $1^{-}, 2^{-}, 3^{-}$, and $\left.4^{-}\right)$, and $\left[\mathrm{V}^{\mathrm{IV}} \mathrm{O}\left(\mathrm{L}^{1-4}\right)(\mathrm{EtOH})\right]$ (Designated by $1^{\prime}, 2^{\prime}, 3^{\prime}$, and $\left.4^{\prime}\right)$ Compounds ${ }^{a}$

$\begin{array}{cccccc}\text { complex (4 mM) } & g_{z} & \begin{array}{c}A_{z}\left(\times 10^{4}\right. \\ \left.\mathrm{cm}^{-1}\right)\end{array} & g_{x}, g_{y} & \begin{array}{c}A_{x}, A_{y}\left(\times 10^{4}\right. \\ \left.\mathrm{cm}^{-1}\right)\end{array} & \begin{array}{c}E_{1 / 2}{ }^{\text {red }} \\ (\mathrm{V})\end{array} \\ \mathbf{1}^{b} & 1.950 & 163.8 & 1.981 & 58.2 & 0.37 \\ \mathbf{1}^{\mathrm{V}} \mathbf{1}^{\mathrm{IV}} \text { (DFT } \\ \text { calculations) }\end{array}$

${ }^{a}$ The column on the right displays the experimental $E_{1 / 2}{ }^{\text {red }}$ (vs SCE) obtained by CV studies. ${ }^{b}$ From EPR spectra recorded after oneelectron reduction for each of the two $\mathrm{V}^{\mathrm{V}}$ centers present in solution.

The chronocoulometric processes were undertaken until the charge supplied reached the value calculated for transfer of half an electron per mole of original complex $(Q=1 / 2 n F c)$; then it was interrupted, and a sample of the electrolyzed solution was collected and frozen for EPR studies.

In accordance with DFT calculations, the lowest unoccupied molecular orbital (LUMO) of the dinuclear $\left[\left\{\mathrm{V}^{\mathrm{V}} \mathrm{O}(\mathrm{L})\right\}_{2}-\mu\right.$-O $]$ compounds $\left[\left\{\mathrm{V}^{\mathrm{V} O}(\text { hnal-abhz })\right\}_{2}-\mu-\mathrm{O}\right]\left(\mathbf{1}^{\mathrm{V}}-\mathbf{1}^{\mathrm{V}}\right)$ and $\left[\left\{\mathrm{V}^{\mathrm{V}} \mathrm{O}\right.\right.$ (han-abhz) $\}_{2}-\mu$-O $]\left(4^{\mathrm{V}}-4^{\mathrm{V}}\right)$ is centered at the $\mathrm{V}$ atoms, oxido ligands, and bridging $\mathrm{O}$ atom (Figure 7$)$. In the singly reduced complex $\left[\mathrm{V}^{\mathrm{V}} \mathrm{O}(\text { hnal-abhz })-\mathrm{O}-\mathrm{V}^{\mathrm{IV}} \mathrm{O}(\text { hnal-abhz })\right]^{-}\left(\mathbf{1}^{\mathrm{V}}-\mathbf{1}^{\mathrm{IV}}\right)$,
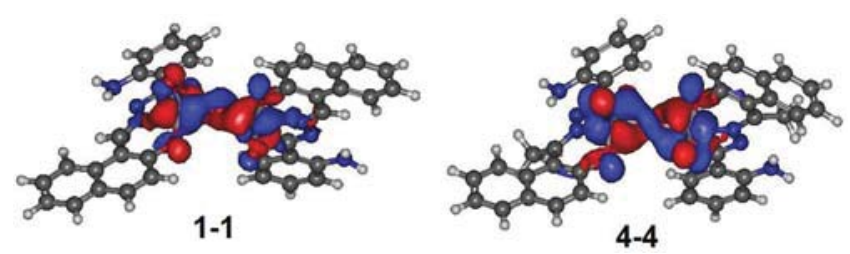

Figure 7. Plots of the LUMO of the $1^{\mathrm{V}}-1^{\mathrm{V}}$ and $4^{\mathrm{V}}-4^{\mathrm{V}}$ complexes. with both unrelaxed and optimized geometries, the spin density is mostly localized at one of the $\mathrm{V}$ centers $(0.95-1.10$ e; Figure 8 ). The negative spin density values at the bridging $\mathrm{O}$ atom and
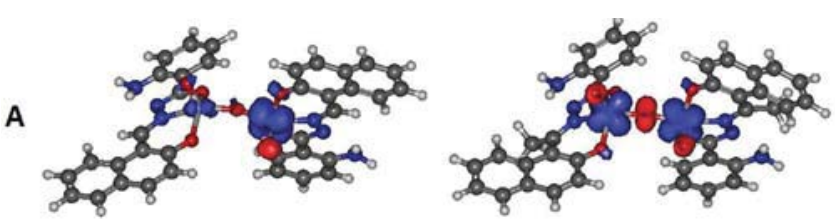

B
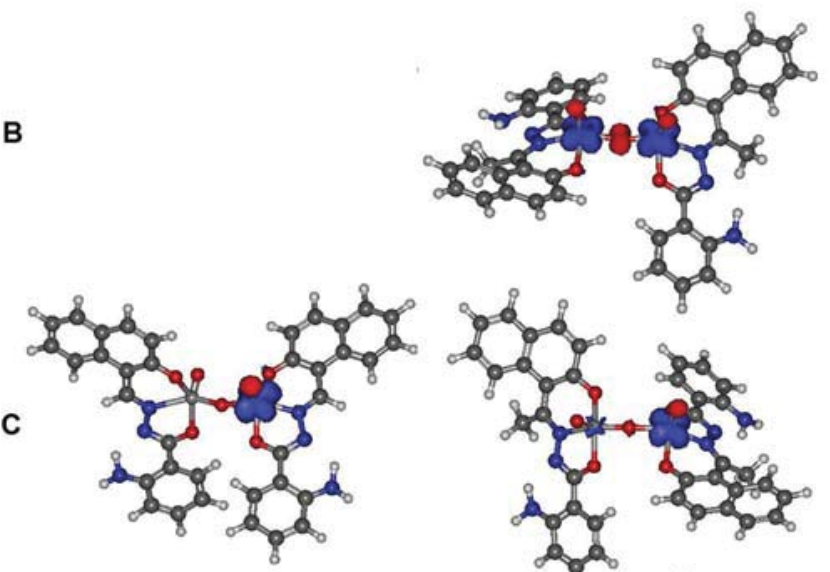

$1^{-}$

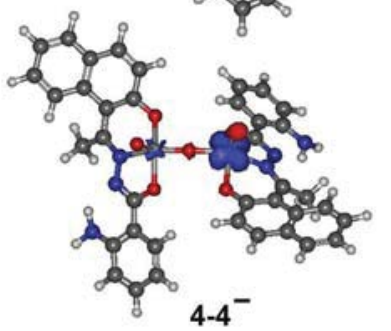

Figure 8. Spin-density distribution in $1^{\mathrm{V}}-1^{\mathrm{IV}}$ and $4 \mathrm{~V}^{\mathrm{V}} 4^{\mathrm{IV}}$ with unrelaxed (A), partially optimized (B), and fully optimized (C) geometries.

oxido ligands $(-0.01$ to -0.11 e) indicate some spin polarization and delocalization between the $\mathrm{V}$ and $\mathrm{O}$ atoms. All of these data suggest that both vertical and adiabatic reductions affect only one metal center and lead to the formation of a mixed-valence complex.

In contrast, in the monoreduced compound [ $\{\mathrm{VO}(\mathrm{han}-$ abhz $\left.)\}_{2}-\mu-\mathrm{O}\right]^{-}\left(4^{\mathrm{V}}-4^{\mathrm{IV}}\right)$ with unrelaxed geometry, the spin density is localized at both metal centers (ca. 0.60 e for each of the $\mathrm{V}$ atoms; Figure 8), indicating that the vertical reduction (i.e., that occurring at the initial moment before the geometry relaxation) equally affects both $\mathrm{V}$ atoms. In the partially optimized structure of $4^{\mathrm{V}}-4^{\mathrm{IV}}$ with the frozen $\mathrm{V}-\mathrm{O}_{\text {bridge }}$ bonds and the $\mathrm{V}-\mathrm{O}-\mathrm{V}$ angle, the spin density is also delocalized between two $\mathrm{V}$ atoms (ca. 0.67 e for each of the $\mathrm{V}$ atoms). However, the fully optimized complex $4^{\mathrm{V}}-4^{\mathrm{IV}}$ corresponds to the mixed-valence species with the spin electron density concentrated at one V center (1.08 e). Therefore, the adiabatic reduction (i.e., that allowing the geometry relaxation) affects only one $\mathrm{V}$ center and leads to the formation of a mixedvalence complex.

These results also indicate that the spin delocalization between two $\mathrm{V}$ atoms is determined by the geometry of the $\mathrm{V}-$ $\mathrm{O}-\mathrm{V}$ fragment. Taking into account that the energy difference between the partially and fully optimized structures of $4^{\mathrm{V}}-4^{\mathrm{IV}}$ is only $2.2 \mathrm{kcal} / \mathrm{mol}$, structural changes leading to delocalization of the spin density between two $\mathrm{V}$ atoms can occur easily in a solution of $4^{\mathrm{V}}-4^{\mathrm{IV}}$ at room temperature due to thermal motion. All of these results correlate with the EPR data, indicating 8-line patterns at both $77 \mathrm{~K}$ and room temperature for $\mathbf{1}^{\mathrm{V}}-\mathbf{1}^{\mathrm{IV}}$, while for $4^{\mathrm{V}}-4^{\mathrm{IV}}$, an 8 -line pattern at $77 \mathrm{~K}$ and a 15 -line pattern at room temperature were obtained.

The EPR spectra of the solutions containing the electroreduced species $\left[\left\{\mathrm{V}^{\mathrm{V}} \mathrm{V}^{\mathrm{IV}} \mathrm{O}_{2}(\mathrm{~L})_{2}\right\}-\mu-\mathrm{O}\right]^{-}$were measured in 
liquid (at room temperature) as well as frozen $(77 \mathrm{~K}) \mathrm{MeCN}$. The EPR spectra of frozen solutions of 3 and 4 after oneelectron electroreduction for each of the two $\mathrm{V}$ centers indicate the presence of (at least) two species in solution. The frozen solution EPR spectra were simulated, ${ }^{19}$ and the spin Hamiltonian parameters thereby obtained are included in Table 4. The DFT calculated values of the ${ }^{51} \mathrm{~V}$ hyperfine coupling constants ${ }^{28}$ are in reasonable agreement with the experimental values (Table 4). Additionally, calculations demonstrate noticeable differences of the $\left|A_{z}\right|$ value for the reduced mononuclear structures (i) $\left[\mathrm{V}^{\mathrm{IV}} \mathrm{O}(\mathrm{OEt})(\mathrm{L})\right]^{-}$ $\left[(156.0-157.4) \times 10^{-4} \mathrm{~cm}^{-1}\right]$ and (ii) $\left[\mathrm{V}^{\mathrm{IV}} \mathrm{O}(\mathrm{L})(\mathrm{EtOH})\right]$ $\left[(163.2-164.4) \times 10^{-4} \mathrm{~cm}^{-1}\right]$ and dinuclear structure $[\{\mathrm{VO}-$ $\left.(\mathrm{L})\}_{2}-\mu-\mathrm{O}\right]^{-}\left[(159.0-160.6) \times 10^{-4} \mathrm{~cm}^{-1}\right]$.

The room temperature $(300 \mathrm{~K}) \mathrm{X}$-band EPR spectra of complexes $\mathbf{1}$ and $\mathbf{2}$ (thus, $\mathbf{1}^{\mathrm{V}}-\mathbf{1}^{\mathrm{IV}}$ and $\mathbf{2}^{\mathrm{V}}-\mathbf{2}^{\mathrm{IV}}$ ) after electroreduction exhibited an 8-line pattern (Figures 9 and S8). In

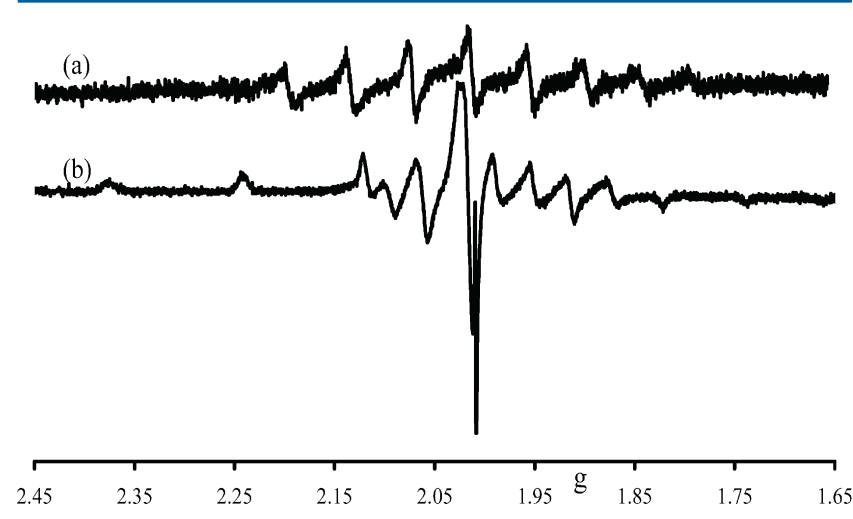

Figure 9. First-derivative EPR spectra of $\mathbf{1}$ in $\mathrm{MeCN}$ after CPE (upon passing the amount of charge corresponding to one-electron reduction for each of the two $\mathrm{V}^{\mathrm{V}}$ centers present): (a) spectrum at room temperature; (b) spectrum of the solution frozen at $77 \mathrm{~K}$. An 8-line pattern is obtained at room temperature. The spin Hamiltonian parameters obtained for the spectrum at $77 \mathrm{~K}$ are in Table 4; the $A_{\text {iso }}$ value for the spectrum at room temperature is $93.2 \times 10^{-4} \mathrm{~cm}^{-1}$.

these cases, the unpaired electron is localized on one of the $\mathrm{V}$ centers. In a frozen solution $(77 \mathrm{~K})$, the EPR spectra also exhibit patterns corresponding to a localized $\mathrm{V}^{\mathrm{IV}} \mathrm{O}$ species. However, in the cases of 3 and 4, the EPR spectra at room temperature exhibit a 15 -line pattern centered around $g=2.00$, with $A_{\text {iso }}$ of ca. 48 and $50 \mathrm{G}$ (Figure 10). This corresponds to the $g$ values and hyperfine pattern anticipated for delocalization of a single, unpaired electron to two equivalent ${ }^{51} \mathrm{~V}$ nuclei $(2 n I$ $+1=15$ for two ${ }^{51} \mathrm{~V}$ centers with $I={ }^{7} / 2$ ). These EPR spectra are indicative of delocalization of the unpaired electron over both metal centers; therefore, the generation of mixed-valence $\mathrm{V}^{\mathrm{IV}}-\mathrm{O}-\mathrm{V}^{\mathrm{V}}$ species is confirmed by EPR. Rather similar EPR spectra were reported by Dinda et al. $^{18 \mathrm{~b}}$ and some other groups. ${ }^{17 \mathrm{c}, \mathrm{o}, 18 \mathrm{a}}$

Interestingly, in the ESI-MS (negative mode) obtained for $1-4$, important peaks with $m / z$ values closely corresponding to one-electron reduction of the dinuclear $\mathbf{M}^{\mathrm{V}}-\mathbf{M}^{\mathrm{V}}$ complexes were detected. This confirms the plausibility and propensity of formation of both the $\mathbf{M}^{\mathrm{V}}-\mathbf{M}^{\mathbf{V}}$ and $\mathbf{M}^{\mathrm{V}}-\mathbf{M}^{\mathrm{IV}}$ species in these systems.

The formation of mixed-valence $\mathrm{V}^{\mathrm{V}}-\mathrm{O}-\mathrm{V}^{\mathrm{IV}}$ species is clearly demonstrated in these experiments for $3^{\mathrm{V}}-3^{\mathrm{IV}}$ and $4^{\mathrm{V}}-4^{\mathrm{IV}}$ for the spectra at $77 \mathrm{~K}$. It is noteworthy that the calculated $A_{z}$ values for the distinct reduced complexes $\mathbf{M}^{\mathrm{V}}-\mathbf{M}^{\mathrm{IV}}, \mathrm{V}^{\mathrm{IV}} \mathrm{O}(\mathrm{OEt})(\mathrm{L})^{-}$ $\left(\mathbf{M}^{-}\right)$, and $\mathrm{V}^{\mathrm{IV}} \mathrm{O}(\mathrm{L})(\mathrm{EtOH})\left(\mathbf{M}^{\prime}\right)$ differ significantly. The observed $A_{z}$ values obtained in the cases of 3 and 4 upon reduction mostly differ from these $A_{z}^{\text {calc }}$ values, with $3^{\prime}$ and $4^{\prime}$ being those that correspond to closer values, while the $A_{z}$ values obtained for $\mathbf{1}$ and $\mathbf{2}$ upon reduction are quite close to those of $\mathbf{1}^{\prime}$ and $\mathbf{2}^{\prime}$ (differences of less than $1 \%$ ).

\section{CONCLUSIONS}

Four substituted hydrazones $\mathrm{H}_{2} \mathrm{~L}^{1-4}$ were prepared by the condensation of aroylhydrazines and naphthol derivatives and characterized. When each of the $\mathrm{H}_{2} \mathrm{~L}^{1-4}$ compounds was reacted with the vanadium $(\mathrm{V})$ precursor $\mathrm{NH}_{4} \mathrm{~V}^{\mathrm{V}} \mathrm{O}_{3}$, the monomeric $\left[\mathrm{V}^{\mathrm{V}} \mathrm{O}(\mathrm{OEt})(\mathrm{L})\right]$ complexes $\mathbf{1 - 4}$ were obtained and characterized in the solid state and in solution by spectroscopic techniques (IR, UV-vis, ${ }^{1} \mathrm{H},{ }^{13} \mathrm{C}$, and ${ }^{51} \mathrm{~V}$ NMR, and ESI-MS). Single-crystal XRD analysis of 1, 3, and 4 confirmed coordination of each of the $\left(\mathrm{L}^{1-4}\right)^{2-}$ ligands with a $\mathrm{O}_{2} \mathrm{~N}$ binding set, each corresponding to a distorted square pyramidal geometry around the $\mathrm{V}^{\mathrm{V}}$ center $[\tau$ values of $0.25(1)$, 0.33 (3), and $0.19(4)]$, where the O,N,O-donor atoms of $\mathbf{L}^{1-4}$ and the $\mathrm{O}$ ethoxido atoms constitute satisfactory $\mathrm{O}_{3} \mathrm{~N}$ basal planes.

The structurally characterized $\left[\mathrm{V}^{\mathrm{V}} \mathrm{O}(\mathrm{OEt})(\mathrm{L})\right]$ compounds transform in solution into the corresponding monooxidobridged divanadium $(\mathrm{V}, \mathrm{V})$ compounds. Formation of the dinuclear $\mu$-oxido species $\left[\left\{\mathrm{V}^{\mathrm{V}} \mathrm{O}\left(\mathrm{L}^{1-4}\right)\right\}_{2}-\mu\right.$-O $]$ generated in situ in solution was confirmed by several spectroscopic techniques (IR and ${ }^{1} \mathrm{H},{ }^{13} \mathrm{C}$, and ${ }^{51} \mathrm{~V} \mathrm{NMR}$ ) as well as by ESI-MS and DFT calculations. For example, in the case of
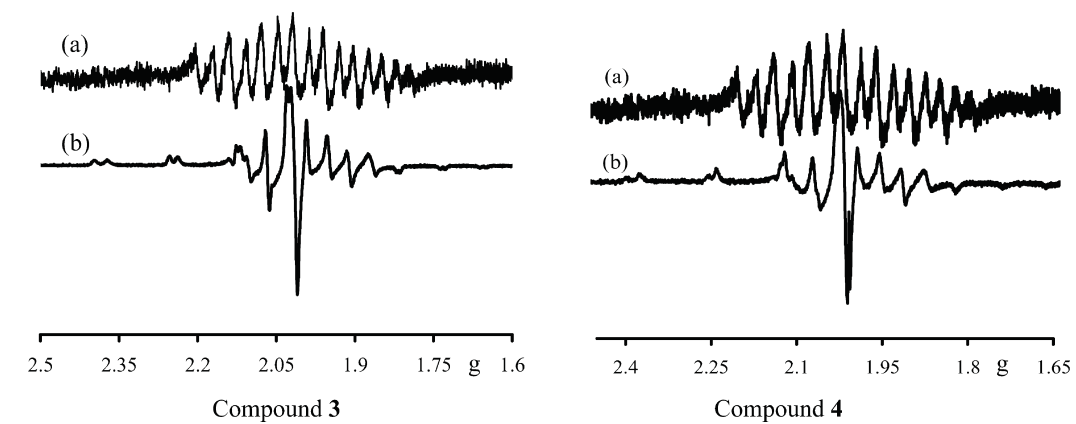

Figure 10. First-derivative EPR spectra measured for solutions of 3 and $\mathbf{4}$ in $\mathrm{MeCN}$ after $\mathrm{CPE}$ (upon passing the amount of charge corresponding to one-electron reduction for each of the two $\mathrm{V}^{\mathrm{V}}$ centers present): (a) spectra at room temperature; (b) spectra of the solutions frozen at $77 \mathrm{~K}$. In both cases, a 15-line pattern is obtained at room temperature. The spin Hamiltonian parameters obtained for the spectra at $77 \mathrm{~K}$ are in Table 4; the $A_{\text {iso }}$ values for the spectra at room temperature are $48 \times 10^{-4} \mathrm{~cm}^{-1}$ (for 3) and $50 \times 10^{-4} \mathrm{~cm}^{-1}$ (for 4). 
compound 4, DFT allowed one to predict that its Gibbs free energy of formation of a dinuclear complex, $2\left[\mathrm{~V}^{\mathrm{V}} \mathrm{O}(\mathrm{OEt})\left(\mathrm{L}^{4}\right)\right]$ $+\mathrm{H}_{2} \mathrm{O} \leftrightarrows\left[\left(\mathrm{V}^{\mathrm{V}} \mathrm{OL}^{4}\right)_{2}-\mu-\mathrm{O}\right]+2 \mathrm{EtOH}$, is only $+2-3 \mathrm{kcal} / \mathrm{mol}$ in a DMSO solution, supporting the proposal that the dinuclear complex $4^{\mathrm{V}}-4^{\mathrm{V}}$ may be easily formed in a DMSO solution in a noticeable amount and that the addition of even a small amount of $\mathrm{EtOH}$ should be enough to shift this equilibrium towards its monomeric form, in agreement with what was observed in NMR $\left({ }^{1} \mathrm{H},{ }^{13} \mathrm{C}\right.$, and $\left.{ }^{51} \mathrm{~V}\right)$ experiments.

The 8-line EPR pattern observed in the EPR spectra at $77 \mathrm{~K}$ of the electrolyzed solutions of 1-4 showed that upon reductive transfer of half an electron per mole to complexes, there was a reduction of $\mathrm{V}^{\mathrm{V}}$ to $\mathrm{V}^{\mathrm{IV}}$. In the cases of the reductions with solutions of 3 and 4, a 15-line EPR pattern was recorded at room temperature, which indicates that the unpaired electron is extensively delocalized between the two $\mathrm{V}$ centers in the formed mixed-valence complex. In contrast, upon similar reductive transfer with solutions of $\mathbf{1}$ and $\mathbf{2}$, an 8 line EPR pattern was obtained at room temperature. It is plausible that mixed-valence compounds $\left[\left\{\operatorname{VO}\left(\mathbf{L}^{1-2}\right)\right\}_{2}-\mu-\mathrm{O}\right]^{-}$ formed but, in these two cases, with the unpaired electron localized on one of the $\mathrm{V}$ atoms. DFT calculations globally allowed rationalization of the data obtained for several systems. Moreover, the DFT-calculated $A_{z}$ values for the proposed $\mathrm{V}^{\mathrm{IV}}$ containing species are in reasonable agreement with the experimental ones.

Further supporting the formation in solution of dinuclear $\mathbf{M}^{\mathrm{V}}-\mathbf{M}^{\mathbf{V}}$ species and their propensity to form $\mathbf{M}^{\mathrm{V}}-\mathbf{M}^{\mathbf{I V}}$ compounds is the detection of peaks in the ESI-MS (negative mode) of $1-4$ with $\mathrm{m} / z$ values very closely matching those expected for oxido-bridged $\mathbf{M}^{\mathrm{V}}-\mathbf{M}^{\mathrm{IV}}$ complexes.

The crystallographic data for the structural analysis of 1, 3, and 4 have been deposited with the Cambridge Crystallographic Data Centre (CCDC 1471661 for 1, CCDC 1471662 for 3, and CCDC 1471663 for 4). A copy of this information may be obtained free of charge from the CCDC, 12 Union Road, Cambridge CB2 1EZ, U.K. [Tel. +44 (0) 1223 762911; e-mail deposit@ccdc.cam.ac.uk].

\section{ASSOCIATED CONTENT}

\section{S Supporting Information}

The Supporting Information is available free of charge on the ACS Publications website at DOI: 10.1021/acs.inorgchem.6b01001.

IR spectra of 3 (Figure S1), UV-vis spectrum of 2 (Figure S2), ${ }^{13} \mathrm{C}$ NMR spectra of complex 1 (Figure S3), ESI-MS spectra of complexes 1, 3, and 4 (Figures S4S6), cyclic voltammograms of 1-4 (Figure S7), EPR spectra of 2 (Figure S8), Cartesian atomic coordinates (Table S1) (PDF)

X-ray crystallographic data in CIF format for 1 (CIF)

$\mathrm{X}$-ray crystallographic data in CIF format for 3 (CIF)

$\mathrm{X}$-ray crystallographic data in CIF format for 4 (CIF)

\section{AUTHOR INFORMATION}

\section{Corresponding Authors}

*E-mail: joao.pessoa@ist.utl.pt (J.C.P.).

*E-mail: rupamdinda@nitrkl.ac.in (R.D.).

\section{Author Contributions}

The manuscript was written through contributions of all authors. All authors have given approval to the final version of the manuscript.

\section{Notes}

The authors declare no competing financial interest.

\section{ACKNOWLEDGMENTS}

Funding for this research was provided by the Department of Science and Technology, India [Grants SR/WOS-A/CS-145/ 2011 (to S.P.D.), SR/FT/CS-016/2008 (to R.D.), and SB/FT/ CS-100/2013 (to A.K.)]. R.D. thanks Prof. H. Reuter, Prof. M. Nethaji, and Prof. S. Chattopadhyay for assistance with XRD analysis. J.C.P. thanks the Portuguese Foundation for Science and Technology [Grants UID/QUI/00100/2013, RECI/QEQQIN/0189/2012, RECI/QEQ-MED/0330/2012, and SFRH/ BPD $/ 90976 / 2012$ (to A.K.)].

\section{REFERENCES}

(1) (a) Rehder, D. Bioinorganic Vanadium Chemistry; John Wiley \& Sons, Ltd.: Chichester, U.K., 2008. (b) Crans, D. C.; Smee, J. J.; Gaidamauskas, E.; Yang, L. Chem. Rev. 2004, 104, 849-902. (c) Pessoa, J. C.; Etcheverry, S.; Gambino, D. Coord. Chem. Rev. 2015, 301-302, 24-48. (d) Costa Pessoa, J.; Garribba, E.; Santos, M. F. A.; SantosSilva, T. Coord. Chem. Rev. 2015, 301-302, 49-86. (e) Kioseoglou, E.; Petanidis, S.; Gabriel, C.; Salifoglou, A. Coord. Chem. Rev. 2015, 301302, 87-105. (f) Rehder, D. Metallomics 2015, 7, 730-742.

(2) McLauchlan, C. C.; Peters, B. J.; Willsky, G. R.; Crans, D. C. Coord. Chem. Rev. 2015, 301-302, 163-199.

(3) Maurya, M. R.; Kumar, A.; Costa Pessoa, J. Coord. Chem. Rev. 2011, 255, 2315-2344.

(4) (a) da Silva, J. A. L.; da Silva, J. J. R. F.; Pombeiro, A. J. L. Coord. Chem. Rev. 2011, 255, 2232-2248. (b) Sutradhar, M.; Martins, L. M. D. R. S.; Guedes da Silva, M. F. C.; Pombeiro, A. J. L. Coord. Chem. Rev. 2015, 301-302, 200-239.

(5) Amadio, E.; Di Lorenzo, R.; Zonta, C.; Licini, G. Coord. Chem. Rev. 2015, 301-302, 147-162.

(6) Pellissier, H. Coord. Chem. Rev. 2015, 284, 93-110.

(7) (a) Noblía, P.; Baran, E. J.; Otero, L.; Draper, P.; Cerecetto, H.; González, M.; Piro, O. E.; Castellano, E. E.; Inohara, T.; Adachi, Y.; Sakurai, H.; Gambino, D. Eur. J. Inorg. Chem. 2004, 2004, 322-328. (b) Gambino, D. Coord. Chem. Rev. 2011, 255, 2193-2203.

(8) (a) Maurya, M. R.; Khan, A. A.; Azam, A.; Kumar, A.; Ranjan, S.; Mondal, N.; Pessoa, J. C. Eur. J. Inorg. Chem. 2009, 2009, 5377-5390. (b) Maurya, M. R.; Khan, A. A.; Azam, A.; Ranjan, S.; Mondal, N.; Kumar, A.; Avecilla, F.; Pessoa, J. C. Dalton Trans. 2010, 39, 13451360. (c) Maurya, M. R.; Agarwal, S.; Abid, M.; Azam, A.; Bader, C.; Ebel, M.; Rehder, D. Dalton Trans. 2006, 937-947. (d) Maurya, M. R.; Kumar, A.; Bhat, A. R.; Azam, A.; Bader, C.; Rehder, D. Inorg. Chem. 2006, 45, 1260-1269. (e) Maurya, M. R.; Kumar, A.; Abid, M.; Azam, A. Inorg. Chim. Acta 2006, 359, 2439-2447. (f) Maurya, M. R; Haldar, C.; Khan, A. A.; Azam, A.; Salahuddin, A.; Kumar, A.; Costa Pessoa, J. Eur. J. Inorg. Chem. 2012, 2012, 2560-2577. (g) Benítez, J.; Cavalcanti de Queiroz, A.; Correia, I.; Alves, M. A.; Alexandre-Moreira, M. S.; Barreiro, E. J.; Lima, L. M.; Varela, J.; González, M.; Cerecetto, H.; Moreno, V.; Pessoa, J. C.; Gambino, D. Eur. J. Med. Chem. 2013, 62, 20-27. (h) Fernández, M.; Varela, J.; Correia, I.; Birriel, E.; Castiglioni, J.; Moreno, V.; Costa Pessoa, J.; Cerecetto, H.; González, M.; Gambino, D. Dalton Trans. 2013, 42, 11900-11911.

(9) Rehder, D.; Costa Pessoa, J.; Geraldes, C.; Castro, M.; Kabanos, T.; Kiss, T.; Meier, B.; Micera, G.; Pettersson, L.; Rangel, M.; Salifoglou, A.; Turel, I.; Wang, D. JBIC, J. Biol. Inorg. Chem. 2002, 7, 384-396.

(10) Dash, S. P.; Pasayat, S.; Bhakat, S.; Roy, S.; Dinda, R.; Tiekink, E. R.; Mukhopadhyay, S.; Bhutia, S. K.; Hardikar, M. R.; Joshi, B. N.; Patil, Y. P.; Nethaji, M. Inorg. Chem. 2013, 52, 14096-14107.

(11) (a) Sutradhar, M.; Pombeiro, A. J. L. Coord. Chem. Rev. 2014, 265, 89-124. (b) Galloni, P.; Conte, V.; Floris, B. Coord. Chem. Rev. 2015, 301-302, 240-299. (c) Honzicek, J.; Vinklárek, J. Inorg. Chim. Acta 2015, 437, 87-94. 
(12) (a) Glas, H.; Herdtweck, E.; Artus, G. R.; Thiel, W. R. Inorg. Chem. 1998, 37, 3644-3646. (b) Dinda, R.; Sengupta, P.; Sutradhar, M.; Mak, T. C. W.; Ghosh, S. Inorg. Chem. 2008, 47, 5634-5640. (c) Li, X.; Lah, M. S.; Pecoraro, V. L. Inorg. Chem. 1988, 27, 46574664. (d) Moon, M.; Pyo, M.; Myoung, Y. C.; Ahn, C. I.; Lah, M. S. Inorg. Chem. 2001, 40, 554-557. (e) Correia, I.; Costa Pessoa, J.; Duarte, M. T.; Henriques, R. T.; Piedade, M. F. M.; Veiros, L. F.; Jakusch, T.; Dornyei, A.; Kiss, T.; Castro, M. M. C. A.; Geraldes, C. F. G. C.; Avecilla, F. Chem. - Eur. J. 2004, 10, 2301-2317. (f) Maurya, M. R.; Uprety, B.; Avecilla, F.; Adao, P.; Costa Pessoa, J. Dalton Trans. 2015, 44, 17736-17755. (g) Maurya, M. R.; Chaudhary, N.; Avecilla, F.; Adão, P.; Costa Pessoa, J. Dalton Trans. 2015, 44, 1211-1232.

(13) (a) Robin, M. B.; Day, P. Adv. Inorg. Chem. Radiochem. 1968, 10, 247-422. (b) Allen, G. C.; Hush, N. S. Prog. Inorg. Chem. 1967, 8, 357-390. (c) Hush, N. S. Prog. Inorg. Chem. 1967, 8, 391-444. (d) Kaim, W.; Lahiri, G. K. Angew. Chem., Int. Ed. 2007, 46, 17781796. (e) Demadis, K. D.; Hartshorn, C. M.; Meyer, T. J. Chem. Rev. 2001, 101, 2655-2686. (f) Kaim, W.; Klein, A.; Glöckle, M. Acc. Chem. Res. 2000, 33, 755-763. (g) D’Alessandro, D. M.; Keene, F. R. Chem. Rev. 2006, 106, 2270-2298. (h) D'Alessandro, D. M.; Keene, F. R. Chem. Soc. Rev. 2006, 35, 424-440. (i) Brunschwig, B. S.; Creutz, C.; Sutin, N. Chem. Soc. Rev. 2002, 31, 168-184. (j) Blondin, G.; Girerd, J. J. Chem. Rev. 1990, 90, 1359-1376. (k) Wilcox, D. E. Chem. Rev. 1996, 96, 2435-2458.

(14) (a) Braun-Sand, S. B.; Wiest, O. J. Phys. Chem. B 2003, 107, 9624-9628. (b) Thomas, J. A. Coord. Chem. Rev. 2013, 257, 15551563.

(15) (a) Young, C. G. Coord. Chem. Rev. 1989, 96, 89-251. (b) Vilas Boas, L. F.; Costa Pessoa, J. In Comprehensive Coordination Chemistry; Wilkinson, G., Gillard, R. D., McCleverty, J. A., Eds.;Pergamon Press: Oxford, U.K., 1987; Vol. 3, pp 453-583. (c) Pessoa, J. C.; Silva, J. A L.; Vieira, A. L.; Vilas Boas, L.; O’Brien, P.; Thornton, P. J. Chem. Soc., Dalton Trans. 1992, 1745-1749. (d) Dutta, S.; Basu, P.; Chakravorty, A. Inorg. Chem. 1993, 32, 5343-5348. (e) Khan, M. I.; Chang, Y. D.; Chen, Q.; Salta, J.; Lee, Y. S.; O’Connor, C. J.; Zubieta, J. Inorg. Chem. 1994, 33, 6340-6350.

(16) Cotton, F. A.; Wilkinson, G. Advanced Inorganic Chemistry, 5th ed.; John Wiley \& Sons: New York, 1988; p 665.

(17) (a) Bhattacharya, K.; Maity, M.; Abtab, S. M. T.; Majee, M. C.; Chaudhury, M. Inorg. Chem. 2013, 52, 9597-9605. (b) Dutta, S. K.; Samanta, S.; Kumar, S. B.; Han, O. H.; Burckel, P.; Pinkerton, A. A.; Chaudhury, M. Inorg. Chem. 1999, 38, 1982-1988. (c) Holwerda, R. A.; Whittlesey, B. R.; Nilges, M. J. Inorg. Chem. 1998, 37, 64-68. (d) Schulz, D.; Weyhermuller, T.; Wieghardt, K.; Nuber, B. Inorg. Chim. Acta 1995, 240, 217-229. (e) Copeland, E. P.; Kahwa, I. A.; Mague, J. T.; McPherson, G. L. J. Chem. Soc., Dalton Trans. 1997, 2849-2852. (f) Dutta, S. K.; Kumar, S. B.; Bhattacharyya, S.; Tiekink, E. R. T.; Chaudhury, M. Inorg. Chem. 1997, 36, 4954-4960. (g) Kojima, A.; Okazaki, K.; Ooi, S.; Saito, K. Inorg. Chem. 1983, 22, 1168-1174. (h) Launay, J.-P.; Jeannin, Y.; Daoudi, M. Inorg. Chem. 1985, 24, 1052-1059. (i) Mahroof-Tahir, M.; Keramidas, A. D.; Goldfarb, R. B.; Anderson, O. P.; Miller, M. M.; Crans, D. C. Inorg. Chem. 1997, 36, 1657-1668. (j) Ghosh, S.; Nanda, K. K.; Addison, A. W.; Butcher, R. J. Inorg. Chem. 2002, 41, 2243-2249. (k) Chen, C.-Y.; Zhou, Z.-H.; Chen, H.-B.; Huang, P.-Q.; Tsai, K.-R.; Chow, Y. L. Inorg. Chem. 2008, 47, 8714-8720. (1) Bino, A.; Cohen, S.; Heitner-Wirguin, C. Inorg. Chem. 1982, 21, 429-431. (m) Sutradhar, M.; Kirillova, M. V.; Guedes da Silva, M. F. C.; Martins, L. M. D. R. S.; Pombeiro, A. J. L. Inorg. Chem. 2012, 51, 11229-11231. (n) Codd, R.; Hambley, T. W.; Lay, P. A. Inorg. Chem. 1995, 34, 877-882. (o) Nishizawa, M.; Hirotsu, K.; Ooi, S.; Saito, K. J. Chem. Soc., Chem. Commun. 1979, 707-708. (p) Chakravarty, J.; Dutta, S.; Chakravorty, A. J. Chem. Soc., Dalton Trans. 1993, 2857-2858. (q) Jin, Y.; Lee, H.-I.; Pyo, M.; Lah, M. S. Dalton Trans. 2005, 797-803. (r) Riechel, T. L.; Sawyer, D. T. Inorg. Chem. 1975, 14, 1869-1875. (s) Mondal, A.; Sarkar, S.; Chopra, D.; Guru Row, T. N.; Pramanik, K.; Rajak, K. K. Inorg. Chem. 2005, 44, 703-708. (t) Zabierowski, P.; Radoń, M.; Szklarzewicz, J.; Nitek, W. Inorg. Chem. Commun. 2014, 41, 72-75.
(18) (a) Mondal, S.; Ghosh, P.; Chakravorty, A. Inorg. Chem. 1997, 36, 59-63. (b) Dinda, R.; Sengupta, P.; Ghosh, S.; Mak, T. C. W. Inorg. Chem. 2002, 41, 1684-1688.

(19) Rockenbauer, A.; Korecz, L. Appl. Magn. Reson. 1996, 10, 2943.

(20) Blanc, E.; Schwarzenbach, D.; Flack, H. D. J. Appl. Crystallogr. 1991, 24, 1035-1041.

(21) Sheldrick, G. M. Acta Crystallogr., Sect. A: Found. Crystallogr. 2008, 64, 112-122.

(22) (a) Becke, A. D. J. Chem. Phys. 1993, 98, 5648-5652. (b) Perdew, J. P. Phys. Rev. B: Condens. Matter Mater. Phys. 1986, 33, 8822-8824. (c) Lee, C.; Yang, W.; Parr, R. G. Phys. Rev. B: Condens. Matter Mater. Phys. 1988, 37, 785-789. (d) Zhao, Y.; Truhlar, D. G. Theor. Chem. Acc. 2008, 120, 215-241. (e) Tao, J. M.; Perdew, J. P.; Staroverov, V. N.; Scuseria, G. E. Phys. Rev. Lett. 2003, 91, 146401.

(23) Frisch, M. J.; Trucks, G. W.; Schlegel, H. B.; Scuseria, G. E.; Robb, M. A.; Cheeseman, J. R.; Scalmani, G.; Barone, V.; Mennucci, B.; Petersson, G. A.; Nakatsuji, H.; Caricato, M.; Li, X.; Hratchian, H. P.; Izmaylov, A. F.; Bloino, J.; Zheng, G.; Sonnenberg, J. L.; Hada, M.; Ehara, M.; Toyota, K.; Fukuda, R.; Hasegawa, J.; Ishida, M.; Nakajima, T.; Honda, Y.; Kitao, O.; Nakai, H.; Vreven, T.; Montgomery, J. A., Jr.; Peralta, J. E.; Ogliaro, F.; Bearpark, M.; Heyd, J. J.; Brothers, E.; Kudin, K. N.; Staroverov, V. N.; Keith, T.; Kobayashi, R.; Normand, J.; Raghavachari, K.; Rendell, A.; Burant, J. C.; Iyengar, S. S.; Tomasi, J.; Cossi, M.; Rega, N.; Millam, J. M.; Klene, M.; Knox, J. E.; Cross, J. B.; Bakken, V.; Adamo, C.; Jaramillo, J.; Gomperts, R.; Stratmann, R. E.; Yazyev, O.; Austin, A. J.; Cammi, R.; Pomelli, C.; Ochterski, J. W.; Martin, R. L.; Morokuma, K.; Zakrzewski, V. G.; Voth, G. A.; Salvador, P.; Dannenberg, J. J.; Dapprich, S.; Daniels, A. D.; Farkas, Ö.; Foresman, J. B.; Ortiz, J. V.; Cioslowski, J.; Fox, D. J. Gaussian09, revision C.01; Gaussian, Inc.: Wallingford, CT, 2010.

(24) (a) Maurya, M. R.; Arya, A.; Kumar, A.; Kuznetsov, M. L.; Avecilla, F.; Costa Pessoa, J. Inorg. Chem. 2010, 49, 6586-6600. (b) Maurya, M. R.; Bisht, M.; Kumar, A.; Kuznetsov, M. L.; Avecilla, F.; Pessoa, J. C. Dalton Trans. 2011, 40, 6968-6983. (c) Maurya, M. R.; Haldar, C.; Kumar, A.; Kuznetsov, M. L.; Avecilla, F.; Costa Pessoa, J. Dalton Trans. 2013, 42, 11941-11962.

(25) (a) Barone, V.; Cossi, M. J. Phys. Chem. A 1998, 102, 19952001. (b) Scalmani, G.; Frisch, M. J. J. Chem. Phys. 2010, 132, 114110-15.

(26) Dolg, M.; Wedig, U.; Stoll, H.; Preuss, H. J. Chem. Phys. 1987, 86, 866-872.

(27) (a) Ditchfield, R. Mol. Phys. 1974, 27, 789-807. (b) Wolinski, K.; Hinton, J. F.; Pulay, P. J. Am. Chem. Soc. 1990, 112, 8251-8260.

(28) (a) Gorelsky, S.; Micera, G.; Garribba, E. Chem. - Eur. J. 2010, 16, 8167-8180. (b) Micera, G.; Garribba, E. Dalton Trans. 2009, 1914-1918. (c) Micera, G.; Garribba, E. J. Comput. Chem. 2011, 32, 2822-2835. (d) Micera, G.; Garribba, E. Eur. J. Inorg. Chem. 2011, 3768-3780. (e) Sanna, D.; Buglyó, P.; Bíró, L.; Micera, G.; Garribba, E. Eur. J. Inorg. Chem. 2012, 2012, 1079-1092. (f) Sanna, D.; Buglyo, P.; Tomaz, A. I.; Pessoa, J. C.; Borovic, S.; Micera, G.; Garribba, E. Dalton Trans. 2012, 41, 12824-12838. (g) Sanna, D.; Bíró, L.; Buglyó, P.; Micera, G.; Garribba, E. J. Inorg. Biochem. 2012, 115, 87-99. (h) Justino, G. C.; Garribba, E.; Pessoa, J. C. JBIC, J. Biol. Inorg. Chem. 2013, 18, 803-813.

(29) Addison, A. W.; Rao, T. N.; Reedijk, J.; van Rijn, J.; Verschoor, G. C. J. Chem. Soc., Dalton Trans. 1984, 1349-1356.

(30) Dash, S. P.; Majumder, S.; Banerjee, A.; Carvalho, M. F. N. N.; Adão, P.; Pessoa, J. C.; Brzezinski, K.; Garribba, E.; Reuter, H.; Dinda, R. Inorg. Chem. 2016, 55, 1165-1182.

(31) Pettersson, L.; Andersson, I.; Gorzsas, A. Coord. Chem. Rev. 2003, 237, 77-87.

(32) Adão, P.; Kuznetsov, M. L.; Barroso, S.; Martins, A. M.; Avecilla, F.; Pessoa, J. C. Inorg. Chem. 2012, 51, 11430-11449.

(33) (a) Tracey, A. S.; Gresser, M. J. Can. J. Chem. 1988, 66, 25702574. (b) Tracey, A. S.; Galeffi, B.; Mahjour, S. Can. J. Chem. 1988, 66, 2294-2298.

(34) (a) Asgedom, G.; Sreedhara, A.; Kivikoski, J.; Valkonen, J.; Kolehmainen, E.; Rao, C. P. Inorg. Chem. 1996, 35, 5674-5683. 
(b) Sun, Y.; Melchior, M.; Summers, D. A.; Thompson, R. C.; Rettig,

S. J.; Orvig, C. Inorg. Chem. 1998, 37, 3119-3121.

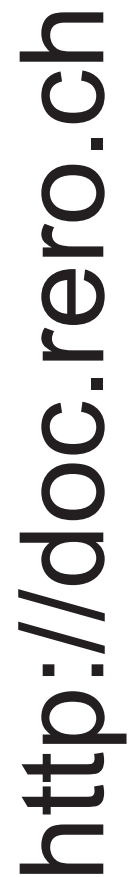

